\title{
Necessary and Sufficient Conditions for Existence and Uniqueness of Recursive Utilities
}

\author{
Jaroslav Borovička and John Stachurski ${ }^{\mathrm{b}}$ \\ a New York University, Federal Reserve Bank of Minneapolis and NBER \\ b Research School of Economics, Australian National University
}

April 24, 2019

\begin{abstract}
We obtain exact necessary and sufficient conditions for existence and uniqueness of solutions of a class of homothetic recursive utility models postulated by Epstein and Zin (1989). The conditions center on a single test value with a natural economic interpretation. The test sheds light on the relationship between valuation of cash flows, impatience, risk adjustment and intertemporal substitution of consumption. We propose two methods to compute the test value when an analytical solution is not available. Several applications are provided.
\end{abstract}

JEL Classifications: D81, G11

Keywords: Recursive preferences, existence, uniqueness

The authors thank Anmol Bhandari, Tim Christensen, Ippei Fujiwara, Jinill Kim, Daisuke Oyama, and Guanlong Ren for useful comments and suggestions. Special thanks are due to Mirosława Zima for her valuable input on local spectral radius conditions. The second author gratefully acknowledges financial support from ARC grant FT160100423. The views expressed herein are those of the authors and not necessarily those of the Federal Reserve Bank of Minneapolis or the Federal Reserve System. 


\section{Disclosure statement for Jaroslav Borovička}

The views expressed in the paper are my own and not necessarily those of the Federal

Reserve Bank of Minneapolis or the Federal Reserve System. I have nothing else to disclose. 
Disclosure statement for John Stachurski

I have nothing to disclose. 


\section{INTRODUCTION}

Recursive preference models such as those discussed in Koopmans (1960), Epstein and Zin (1989) and Weil (1990) play an important role in macroeconomic and financial modeling. For example, the long-run risk models analyzed in Bansal and Yaron (2004), Hansen et al. (2008), Bansal et al. (2012) and Schorfheide et al. (2018) have employed such preferences in discrete time infinite horizon settings with a variety of consumption path specifications to help resolve long-standing empirical puzzles identified in the literature.

In recursive utility models, the lifetime value of a consumption stream from a given point in time is expressed as the solution to a nonlinear forward-looking equation. While this representation is convenient and intuitive, it can also be vacuous, in the sense that no finite solution to the forward looking recursion exists. Moreover, even when a solution is found, this solution lacks predictive content unless some form of uniqueness can also be established. In general, identifying restrictions that imply existence and uniqueness of a solution for an empirically relevant class of consumption streams is challenging.

The aim of the present paper is to obtain existence and uniqueness results that are as tight as possible in a range of empirically plausible settings, while restricting attention to practical conditions that can be tested in applied work. To this end, we provide conditions for existence and uniqueness of solutions to the class of homothetic preferences studied in Epstein and Zin (1989), while admitting both stationary and nonstationary consumption paths. These conditions are both necessary and sufficient, and hence as tight as possible in the setting we consider. In particular, if the conditions hold then a unique, globally attracting solution exists, while if not then no finite solution exists. Existence of a finite solution is equivalent to the existence of a finite wealth-consumption ratio, a central object of interest in asset pricing.

To give more detail on that setting, let preferences be defined recursively by

$$
V_{t}=\left[(1-\beta) C_{t}^{1-1 / \psi}+\beta\left\{\mathcal{R}_{t}\left(V_{t+1}\right)\right\}^{1-1 / \psi}\right]^{1 /(1-1 / \psi)},
$$

where $\left\{C_{t}\right\}$ is a consumption path, $V_{t}$ is the utility value of the path extending on from time $t$ and $\mathcal{R}_{t}$ is the Kreps-Porteus certainty equivalent operator

$$
\mathcal{R}_{t}\left(V_{t+1}\right)=\left(\mathbb{E}_{t} V_{t+1}^{1-\gamma}\right)^{1 /(1-\gamma)}
$$

The parameter $\beta \in(0,1)$ is a time discount factor, while $\gamma \neq 1$ governs risk aversion and $\psi \neq 1$ is the elasticity of intertemporal substitution. We take the consumption stream as given and seek a solution for normalized utility $V_{t} / C_{t}$. 
The first step in our approach is to associate to each consumption process the risk-adjusted long-run mean consumption growth rate

$$
\mathcal{M}_{C}:=\lim _{n \rightarrow \infty}\left[\mathcal{R}\left(\frac{C_{n}}{C_{0}}\right)\right]^{1 / n},
$$

where $\mathcal{R}$ is the unconditional version of the Kreps-Porteus certainty equivalent operator. Beginning with the case where the state vector driving the conditional distribution of consumption growth takes values in a compact set - which is where the sharpest results can be obtained - we show that a unique solution exists if and only if $\Lambda<1$, where

$$
\Lambda:=\beta \mathcal{M}_{C}^{1-1 / \psi}
$$

Under the same compactness restriction, we also show that the condition $\Lambda<1$ is both necessary and sufficient for global convergence of successive approximations associated with a natural fixed point mapping. In fact our results establish that convergence of successive approximations itself implies that a unique solution exists, and that the limit produced through this process is equal to the solution. Furthermore, we prove that when the condition $\Lambda<1$ fails, not only does existence and uniqueness of a solution fail, but existence fails specifically.

The value $\Lambda$ represents the risk-adjusted long-term consumption growth rate modified by impatience and the intertemporal substitutability of consumption. Despite the fact that the preference recursion (1) intertwines the contributions of impatience, intratemporal risk aversion and intertemporal elasticity of substitution to value, the condition $\Lambda<1$ effectively separates these forces. Details of the consumption growth process, such as its persistence or higher moments of its innovations, matter only through the long-run distribution of consumption growth encoded in $\mathcal{M}_{C}$. Additional discussion of the intuition behind condition (4) is provided in section 3 and the applications.

In addition to the preceding results, we use a local spectral theorem to show that

$$
\mathcal{M}_{C}=r(K)^{1 /(1-\gamma)}
$$

where $r(K)$ is the spectral radius of a valuation operator $K$ determined by the primitives and clarified below. This result is useful on two levels. First, spectral radii and dominant eigenfunctions associated with valuation operators have increasingly been used to understand long-run risks and long-run values in macroeconomic and financial applications by inducing a decomposition of the stochastic discount factor (see, e.g., Alvarez and Jermann (2005); Hansen and Scheinkman (2009); Qin and Linetsky (2017); Christensen (2017)). The identification in (5) allows us to connect to and draw insights from this literature. Second, on a computational level, when the state space for the state process is finite, the 
valuation operator $K$ is just a matrix, and the spectral radius is easily computed. From this one can compute the test statistic $\Lambda$ via (5). When the state space is not finite, one can still implement this idea after discretization.

When the state space is high dimensional, accurate discretization is nontrivial and calculation of the spectral radius becomes computationally expensive. For these scenarios, we propose instead a Monte Carlo method to calculate the test value $\Lambda$, based around the idea of simulating consumption paths from a given specification and calculating the risk-adjusted expectation on the right hand side of (3) by averaging over these paths. This approach is straightforward to implement and relatively insensitive to the dimension of the state space. Another advantage is that the routine is easily be parallelized by simulating independent consumption paths along multiple execution threads.

All of the theoretical results on existence, uniqueness and convergence of successive approximations discussed above are stated in the context of a compact-valued state process, which drives the persistent component of consumption growth. In this setting we apply a fixed point theorem due to Du (1990), which extends to abstract vector space the idea that an increasing concave function $f$ from $\mathbb{R}_{+}$to itself has at most one strictly positive fixed point - and at least one such fixed point if the slope conditions $f^{\prime}(0)>1$ and $f^{\prime}(\infty)<1$ are satisfied. In the case of the valuation problem considered in this paper, monotonicity and concavity are inherent in the preference specification, while the condition $\Lambda<1$ is the key to the slope conditions. The arguments required for the last step are nontrivial and compactness of the state space plays a significant role.

At the same time, we do provide some guidance on the implications of the condition $\Lambda<1$ when the state space is unbounded. In this setting we show that, under an auxiliary technical restriction related to compactness of iterates of the operator $K$, the condition $\Lambda<1$ is again necessary and sufficient for existence of a solution. As before, convergence of successive approximations to a given function implies that the limiting function is a solution, and this can only occur when $\Lambda<1$. The identification in (5) continues to be valid when these conditions hold, and the Monte Carlo method described above can still be applied. The proofs use an approximation argument that bootstraps previously stated results from the compact case.

We provide a series of applications of the numerical and theoretical results in section 4 . We start with the model of a trend-stationary consumption process from Alvarez and Jermann (2005), in which we can characterize our condition analytically. The result reveals that transitory uncertainty in the consumption process, such as stationary fluctuations around a deterministic time trend, are immaterial for the existence of the continuation value. 
Next, we focus on a frequently used case in which the dynamics of conditional moments of consumption growth are encoded using a Markov chain. Specifically, we use the calibration from Johannes et al. (2016) and consider two information structures, one in which the agent observes the realizations of the Markov chain and another in which the agent must learn about the underlying state from consumption growth realizations that reveal the state only imperfectly. In the former case, given the simple structure of the state space, evaluating the test condition involves computing the spectral radius in (5) as the largest eigenvalue of a small matrix. In the latter case, the state space is continuous and encodes subjective probabilities of unobserved states of the Markov chain, updated using Bayes rule. Despite the fact that the state space and transition dynamics are now much more complex, our results reveal that the continuation value exists exactly for the same set of parameters as in the full information case. The underlying reason is the transitory role of state uncertainty for the conditional distribution of future consumption growth that has no impact on the value of $\mathcal{M}_{C}$ in (3).

Our main quantitative application is the long-run risk model specified in Schorfheide et al. (2018). Existence of finite continuation values is a nontrivial issue in such models because (i) discounting is extremely small in order to raise the importance of persistent risk components, pushing them towards the boundary between stability and instability, and (ii) the state dynamics are nonlinear and relatively high dimensional. Using numerical methods, we show that the condition $\Lambda<1$ is satisfied with arbitrarily small amounts of truncation and almost certainly satisfied in the original model without truncation. We also provide several robustness checks that relate alternative approximation procedures and the impact of truncation.

Finally, we show that our results can also be applied to production economies where consumption is endogenously determined. In many applications, consumption is cointegrated with an exogenously specified process that drives uncertainty in the model. Since transitory fluctuations in the consumption process are irrelevant, the risk-adjusted long-run growth rate can be directly computed using the exogenous driving process, without the knowledge of specific details of the consumption process.

Regarding existing literature, sufficient conditions for existence and uniqueness of recursive utilities were provided by Epstein and Zin (1989) and Marinacci and Montrucchio (2010). These conditions require a finite bound $B_{c}$ on consumption growth $C_{t+1} / C_{t}$ that holds asymptotically with probability one. As a result, they cannot be applied to many recent specifications of the consumption processes, such as the long-run risk specification given in section 4.4, as consumption growth in those settings is unbounded above. Even 
if a finite bound is obtained by truncation of the shocks, we show that the resulting conditions are always stricter than the ones presented in this paper, and typically far too strict for realistic parameterizations. This is due to the fact that probability one bounds restrict utility uniformly along every future consumption trajectory, while the results in this paper consider what happens on average across all paths. In other words, our results are sharper because recursive utility specifications, while nonlinear, are still defined using integration over future continuation values. Conversely, focusing only on the upper tail of the consumption growth process leads to excessively tight stability conditions.

Another condition for existence of recursive utilities can be found in Alvarez and Jermann (2005), which focuses on the case where consumption has a deterministic time trend. Our condition $\Lambda<1$ is also weaker than their condition, as shown in section 5 . The intuition behind this is that Alvarez and Jermann (2005) use a fixed point argument that requires contraction in one step. In contrast, the restriction $\Lambda<1$ is an asymptotic condition that ignores short-run fluctuations in consumption.

Also related is Hansen and Scheinkman (2012), who study Epstein-Zin utility models with unbounded consumption growth. Their approach is to connect the solution to the Epstein-Zin utility recursion and the Perron-Frobenius eigenvalue problem associated with a linear operator, denoted in their paper by $\mathbb{T}$, that is proportional to the operator $K$ discussed above. Consumption growth is a function of an unbounded exogenous state process. In this setting they show that a solution exists when a joint restriction holds on the spectral radius of $\mathbb{T}$ and the preference parameters, along with integrability conditions on the eigenfunctions of $\mathbb{T}$ already mentioned. They also obtain a uniqueness result for some parameter values (although not the most empirically relevant ones).

For the case where $\mathbb{X}$ is compact, our approach has the following advantages: First, we obtain uniqueness of the solution for all parameterizations. Second, we show our conditions are necessary as well as sufficient, both for existence and for uniqueness. Third, we obtain a globally convergent method of computation, and show that it converges if and only if a solution exists. Fourth, we provide multiple representations of the test value, strengthening the economic interpretation, as well as a method of computation that can be applied in high dimensional settings. Fifth, we avoid the auxiliary conditions in Hansen and Scheinkman (2012) involving integrability restrictions on the eigenfunctions of the operator $\mathbb{T}$, which means that all our conditions are straightforward to test in applied settings. For the case where $\mathbb{X}$ is not compact, our results also serve to augment those of Hansen and Scheinkman (2012) by showing that the condition $\Lambda<1$ is necessary as well as sufficient for existence of a solution. 
In another related study, Guo and He (2018) consider an extension to the Epstein-Zin recursive utility model that includes utility measures for investment gains and losses. As a part of that study they obtain results for existence, uniqueness and convergence of solutions to Epstein-Zin recursive utility models with consumption specifications analogous to those in Hansen and Scheinkman (2012), except that the state space is restricted to be finite. In comparison, we allow for the state space to be countably or uncountably infinite and we establish not just sufficiency but also necessity.

The paper is structured as follows: Section 2 considers the risk-adjusted long-run mean consumption growth rate in more depth. Section 3 states our main results. Section 4 discusses applications. Section 5 contrasts our results with alternative sufficient conditions in the previous literature. Section 6 treats the unbounded case and section 7 concludes. All proofs are deferred to the appendix. ${ }^{1}$

\section{Consumption Paths and Risk-Adjusted Growth}

Before stating our main results, we introduce our baseline model for consumption paths and address an important issue: Since $\Lambda=\beta \mathcal{M}_{C}^{1-1 / \psi}$, the practicality of our condition $\Lambda<1$ depends on the ability to accurately evaluate the risk-adjusted long-run mean consumption growth rate $\mathcal{M}_{C}$, as defined in (3). For some specifications of the consumption path, an analytical expression for $\mathcal{M}_{C}$ exists. For others, however, no such expression can be obtained. In this second case, we must turn to numerical methods to evaluate $\mathcal{M}_{C}$. This section discusses two methods to compute $\mathcal{M}_{C}$ numerically. The main aims of this section are to (a) build intuition on $\mathcal{M}_{C}$ by treating some relatively simple cases and (b) provide evidence affirming that $\mathcal{M}_{C}$ and hence $\Lambda$ can be evaluated sufficiently accurately even when no closed form solution exists.

2.1. Consumption Paths. As in Hansen and Scheinkman (2012), we suppose that consumption growth has the generic specification

$$
\ln \left(C_{t+1} / C_{t}\right)=\kappa\left(X_{t}, X_{t+1}, \varepsilon_{t+1}\right),
$$

where $\kappa$ is a continuous function, $\left\{X_{t}\right\}$ is an exogenous state process and $\left\{\varepsilon_{t}\right\}$ is an IID innovation process supported on $\mathbb{Y} \subset \mathbb{R}^{k}$ and independent of $\left\{X_{t}\right\}$. The state process is assumed to be stationary and Markov, taking values in a subset $\mathbb{X}$ of $\mathbb{R}^{n}$. The unconditional density of each $X_{t}$ is denoted by $\pi$. The function $q(x, \cdot)$ represents the conditional density of $X_{t+1}$ given $X_{t}=x$. All of our results include the case where $\mathbb{X}$ is finite and,

\footnotetext{
${ }^{1}$ The repository https://github.com/jstac/recursive_utility_code contains code that replicates all of our numerical results.
} 
in this case, the transition density $q(x, y)$ should be interpreted as a transition matrix. More generally, the term "density" should be understood as a synonym for "probability mass function."

Assumption 2.1. The function $q$ is continuous and the $\ell$-step transition density $q^{\ell}$ is everywhere positive at some $\ell \in \mathbb{N}$.

Continuity can be ignored when $\mathbb{X}$ is finite. Positivity of $q^{\ell}$ for some $\ell$ means that $\left\{X_{t}\right\}$ is both aperiodic and irreducible, guaranteeing uniqueness of the stationary distribution $\pi$ and providing regularity for asymptotic values such as the the risk-adjusted long-run mean consumption growth rate. Assumption 2.1 is either satisfied directly in our applications or can be validated after an arbitrarily small perturbation (as is the case for the learning application-see section 4.3).

For example, consider the consumption growth specification

$$
\begin{aligned}
\ln \left(C_{t+1} / C_{t}\right) & =\mu_{c}+X_{t}+\sigma_{c} \varepsilon_{t+1} \\
X_{t+1} & =\rho X_{t}+\sigma \eta_{t+1}
\end{aligned}
$$

from section I.A of Bansal and Yaron (2004). Here $-1<\rho<1$ and $\left\{\varepsilon_{t}\right\}$ and $\left\{\eta_{t}\right\}$ are IID standard normal innovations. ${ }^{2}$ With the state process set to $\left\{X_{t}\right\}$, we have $q(x, \cdot)=$ $N\left(\rho x, \sigma^{2}\right)$ and assumption 2.1 is satisfied with $\ell=1$ whenever $\sigma>0$.

For this model of consumption dynamics, an analytical expression for $\mathcal{M}_{C}$ exists, even though the same is not true for the continuation value $V_{t}$. To see this, observe that $C_{n} / C_{0}=\exp \left(n \mu_{c}+\sum_{t=1}^{n} H_{t}\right)$ where $H_{t}:=X_{t}+\sigma_{c} \varepsilon_{t}$, so the risk-adjusted long-run mean consumption growth rate is

$$
\mathcal{M}_{C}=\lim _{n \rightarrow \infty}\left\{\mathcal{R} \exp \left(n \mu_{c}+\sum_{t=1}^{n} H_{t}\right)\right\}^{1 / n}
$$

Here $\mathcal{R}$ is the unconditional Kreps-Porteus certainty equivalent operator defined by $\mathcal{R}[Y]:=\mathbb{E}\left[Y^{1-\gamma}\right]^{1 /(1-\gamma)}$. Noting that $\left\{H_{t}\right\}$ is Gaussian and setting $s_{n}$ equal to the variance of $\sum_{t=1}^{n} H_{t}$, we have

$$
\mathcal{R} \exp \left(n \mu_{c}+\sum_{t=1}^{n} H_{t}\right)=\exp \left\{n \mu_{c}+\frac{(1-\gamma) s_{n}^{2}}{2}\right\} .
$$

\footnotetext{
${ }^{2}$ These calculations can be further extended to the case where consumption growth is a component of a VAR, as in Hansen et al. (2008) or Bansal et al. (2014).
} 
Using the fact that $\sum_{t=1}^{n} H_{t}$ is the sum of the independent terms $\sum_{t=1}^{n} X_{t}$ and $\sigma_{c} \sum_{t=1}^{n} \varepsilon_{t}$, along with the $\operatorname{AR}(1)$ dynamics in (8), straightforward calculations lead to

$$
s_{n}^{2}=n \sigma_{c}+\frac{\sigma^{2}}{1-\rho^{2}}\left\{n+2(n-1) \frac{\rho}{1-\rho}-2 \rho^{2} \frac{1-\rho^{n-1}}{(1-\rho)^{2}}\right\} .
$$

Raising the right hand side of (9) to the power of $1 / n$ and taking the limit yields

$$
\mathcal{M}_{C}=\exp \left\{\mu_{c}+\frac{1}{2}(1-\gamma)\left(\sigma_{c}^{2}+\frac{\sigma^{2}}{(1-\rho)^{2}}\right)\right\} .
$$

While the unconditional variance of the one-period consumption growth rate is $\sigma_{c}+$ $\sigma^{2} /\left(1-\rho^{2}\right)$, persistence in consumption growth implies that the long-run variance of the consumption growth rate is given by $\sigma_{c}+\sigma^{2} /(1-\rho)^{2}$. Typical calibrations of risk aversion set $\gamma>1$ and higher persistence and volatility then contribute negatively to $\mathcal{M}_{C}$. For example, under the parameterization of the consumption process in table I of Bansal and Yaron (2004), the expression in (10) evaluates to 1.00045 when $\gamma=7.5$ and 0.99964 when $\gamma=12.5^{3}$

2.2. The Finite State Case. The finite state setting gives us an alternative view on the risk-adjusted long-run mean consumption growth rate $\mathcal{M}_{C}$ and an alternative way to compute it. In particular, if $\mathbb{X}$ is finite with typical elements $x, y$ and $K$ is the matrix with $(x, y)$-th element

$$
K(x, y):=\sum_{y \in \mathbb{X}} \int \exp [(1-\gamma) \kappa(x, y, \varepsilon)] \nu(\mathrm{d} \varepsilon) q(x, y),
$$

then (5) holds; that is,

$$
\mathcal{M}_{C}=r(K)^{1 /(1-\gamma)} \quad \text { when } \quad r(K)=\max _{\lambda \in E}|\lambda| .
$$

Here $E$ is the set of eigenvalues of $K$, so $r(K)$ is the spectral radius of the matrix $K$.

To gain some understanding as to why the alternative representation of $\mathcal{M}_{C}$ in (12) is valid, note that, for all $x$ in $\mathbb{X}$ and all $n$ in $\mathbb{N}$, we have

$$
K^{n} \mathbb{1}(x)=\mathbb{E}_{x} \exp \left\{(1-\gamma) \sum_{t=1}^{n} \kappa\left(X_{t-1}, X_{t}, \varepsilon_{t}\right)\right\} .
$$

Here $K^{n}$ is the $n$-th power of $K$ in (11) and $\mathbb{1}$ is a vector of ones. The expectation $\mathbb{E}_{x}$ conditions on $X_{0}=x$. That the identity in (13) is true at $n=1$ follows immediately from the definition of $K$ in (11), and the case of general $n$ can be confirmed by induction. Let

\footnotetext{
${ }^{3}$ The parameter values in question are $\mu_{c}=0.0015, \rho=0.979, \sigma=0.00034$ and $\sigma_{c}=0.0078$.
} 
$\|\cdot\|$ be the $L_{1}$ vector norm defined by $\|h\|=\sum_{x \in \mathbb{X}}|h(x)| \pi(x)$. By (13) and the Law of Iterated Expectations, we have

$$
\left\|K^{n} \mathbb{1}\right\|=\sum_{x \in \mathbb{X}} K^{n} \mathbb{1}(x) \pi(x)=\mathbb{E} \exp \left\{(1-\gamma) \sum_{t=1}^{n} \kappa\left(X_{t-1}, X_{t}, \varepsilon_{t}\right)\right\} .
$$

In other words,

$$
\left\|K^{n} \mathbb{1}\right\|=\mathbb{E}\left(\frac{C_{n}}{C_{0}}\right)^{1-\gamma}=\left[\mathcal{R}\left(\frac{C_{n}}{C_{0}}\right)\right]^{1-\gamma} .
$$

Gelfand's formula for the spectral radius tells us that $\left\|K^{n}\right\|^{1 / n} \rightarrow r(K)$ as $n \rightarrow \infty$ whenever $\|\cdot\|$ is a matrix norm, and this result can, in the present context, be modified to show that $\left\|K^{n} \mathbb{1}\right\|^{1 / n} \rightarrow r(K)$ also holds. ${ }^{4}$ Connecting the last result with (15) gives

$$
\mathcal{M}_{C}^{1-\gamma}=\lim _{n \rightarrow \infty}\left[\mathcal{R}\left(\frac{C_{n}}{C_{0}}\right)\right]^{\frac{1-\gamma}{n}}=\lim _{n \rightarrow \infty}\left\|K^{n} \mathbb{1}\right\|^{1 / n}=r(K)
$$

which justifies the claim in (12). In several applications below we use this identity by calculating $r(K)$ numerically and then recovering $\mathcal{M}_{C}$ via $\mathcal{M}_{C}=r(K)^{1 /(1-\gamma)}$.

2.3. Discretization. As discussed in the previous section, if the state space is finite, then we can use the identity $\mathcal{M}_{C}=r(K)^{1 /(1-\gamma)}$ obtained in (16) to calculate $\mathcal{M}_{C}$, which in turn allows us to compute the stability exponent $\Lambda$. If, on the other hand, $\mathbb{X}$ is not finite, then one option is to discretize the model, leading to a finite state space and a finite set of transition probabilities $q(x, y)$, and then proceed as above. Here we investigate the accuracy of this procedure.

Our experiment is based on the Bansal-Yaron consumption growth dynamics in (7)(8), where an analytical expression for $\mathcal{M}_{C}$ was obtained in (10). We first discretize the Gaussian AR(1) state process (8) using Rouwenhorst's method (Rouwenhorst, 1995). Then we compute the matrix $K$ in (11) corresponding to this discretized state process, calculate the spectral radius $r(K)$ using linear algebra routines and, from there, compute the associated value for $\mathcal{M}_{C}$ as $r(K)^{1 /(1-\gamma)}$. Finally, we compare the result with the true value of $\mathcal{M}_{C}$ obtained from the analytical expression (10).

Table 1 shows such a comparison at a range of parameter values and levels of discretization. The preference parameter $\gamma$ is varied across the rows, as shown in the first column, while the remaining parameters are sourced from table I of Bansal and Yaron (2004), as in footnote 3 . The second column shows the true value of $\mathcal{M}_{C}$ for the nondiscretized model. The remaining columns show the value computed using the numerical procedure

\footnotetext{
${ }^{4}$ This is a finite dimensional version of the local spectral radius result discussed in the introduction. A complete proof that extends to infinite state settings is given in theorem A.1 of the appendix.
} 


\begin{tabular}{l|c|cccc}
\hline & true value & $D=5$ & $D=50$ & $D=100$ & $D=200$ \\
\hline$\gamma=7.5$ & 1.0004504 & 1.0004998 & 1.0004549 & 1.0004527 & 1.0004516 \\
$\gamma=10.0$ & 1.0000466 & 1.0001658 & 1.0000584 & 1.0000525 & 1.0000496 \\
$\gamma=12.5$ & 0.9996430 & 0.9998662 & 0.9996673 & 0.9996552 & 0.9996491 \\
\hline
\end{tabular}

TABLE 1. True value and discrete approximation of $\mathcal{M}_{C}$, Bansal-Yaron model

discussed in the previous paragraph at different levels of discretization. For example, $D=5$ means that the $\mathrm{AR}(1)$ process (8) was discretized into a 5 state Markov chain using the Rouwenhorst method. The results show that the discretization based method is accurate for this model, even for relatively coase approximations.

2.4. A Monte Carlo Method. One potential issue with the discretization based method discussed in section 2.3 is that the algorithm is computationally inefficient when the state space is large. For this reason we also propose a Monte Carlo method to calculate an approximation to $\mathcal{M}_{C}$ that is less susceptible to the curse of dimensionality. The first step is to replace the limit in the definition of $\mathcal{M}_{C}$ by some finite but large $n$, which leads to

$$
\mathcal{M}_{C}(n):=\left\{\mathbb{E}\left(\frac{C_{n}}{C_{0}}\right)^{1-\gamma}\right\}^{\frac{1}{1-\gamma} \frac{1}{n}}
$$

Next, the expectation in (17) is replaced by a sample average over $m$ independent consumption paths, generated according to the specifications of the model in question. In particular, with $\left\{C_{t}^{(j)}\right\}$ as the $j$-th of the $m$ consumption paths, ${ }^{5}$ we take the approximation

$$
\mathcal{M}_{C}(m, n):=\left\{\frac{1}{m} \sum_{j=1}^{m}\left(\frac{C_{n}^{(j)}}{C_{0}^{(j)}}\right)^{1-\gamma}\right\}^{\frac{1}{1-\gamma} \frac{1}{n}}
$$

With fixed $n$ and $Y^{(j)}:=\left(C_{n}^{(j)} / C_{0}^{(j)}\right)^{1-\gamma}$, the Strong Law of Large Numbers yields $\frac{1}{m} \sum_{j=1}^{m} Y^{(j)} \rightarrow \mathbb{E} Y$ as $m \rightarrow \infty$ with probability one. However, this result is only asymptotic and our main concern is whether the estimator $\mathcal{M}_{C}(m, n)$ has good properties when $m$ and $n$ are moderate. To test this, we again use the lognormal consumption model and compare our approximations of $\mathcal{M}_{C}$ with the true value obtained via the analytical expression given in (10).

\footnotetext{
${ }^{5}$ Since $\mathbb{E}$ in $(17)$ is the unconditional expectation, we draw the initial state $X_{0}^{(j)}$ from its stationary distribution when computing $\left\{C_{t}^{(j)}\right\}$.
} 


\begin{tabular}{l|rrrrr}
\hline & $m=1000$ & $m=2000$ & $m=3000$ & $m=4000$ & $m=5000$ \\
\hline$n=250$ & 1.0006940 & 1.0006905 & 1.0006940 & 1.0006932 & 1.0006934 \\
& $(0.000076)$ & $(0.000048)$ & $(0.000032)$ & $(0.000037)$ & $(0.000029)$ \\
$n=500$ & 1.0006208 & 1.0006091 & 1.0005813 & 1.0005733 & 1.0005775 \\
& $(0.000084)$ & $(0.000066)$ & $(0.000068)$ & $(0.000084)$ & $(0.000062)$ \\
$n=750$ & 1.0005979 & 1.0005762 & 1.0005764 & 1.0005611 & 1.0005523 \\
& $(0.000112)$ & $(0.000096)$ & $(0.000076)$ & $(0.000074)$ & $(0.000092)$ \\
\hline
\end{tabular}

TABLE 2. Realizations of $\mathcal{M}_{C}(m, n)$ when $\mathcal{M}_{C}=1.0004504$. Values shown are mean and standard deviation over 1,000 independent draws.

Table 2 illustrates our results. The consumption path parameters are again chosen to match Bansal and Yaron (2004), as in footnote 3. The parameter $\gamma$ is set to 7.5, which matches the first row of table 1 . The true value of $\mathcal{M}_{C}$ calculated from the analytical expression (10) is 1.0004504, as shown in the caption for the table. The interpretation of $n$ and $m$ in the table is consistent with the right hand side of (18). For each $n, m$ pair, we compute $\mathcal{M}_{C}(n, m)$ a total of 1,000 times using independent draws, and then present the mean and the standard deviation of the sample in the corresponding table cell. The Monte Carlo approximation is accurate up to three decimal places in all simulations. Standard deviations are small and decline with $m .^{6}$

High accuracy in estimating $\mathcal{M}_{C}$ translates into similarly high accuracy in estimating $\Lambda=\beta \mathcal{M}_{C}^{1-1 / \psi}$ once we introduce the additional preference parameters $\beta$ and $\psi$. Table 3 illustrates this point. Each $(m, n)$ cell in the table gives the mean and standard deviation of 1,000 draws of

$$
\Lambda(m, n):=\beta \mathcal{M}_{C}(m, n)^{1-1 / \psi} .
$$

The draws of $\mathcal{M}_{C}(m, n)$ are computed as in table 2, while the remaining parameters are set to $\beta=0.998$ and $\psi=1.5$, as in Bansal and Yaron (2004). The true value $\Lambda=0.9981498$ from the caption of table 3 is calculated as $\Lambda=\beta \mathcal{M}_{C}^{1-1 / \psi}$ where $\mathcal{M}_{C}$ is obtained from the analytical expression (10). The significance of the actual values is discussed below.

\section{Existence and Uniqueness of Recursive Utilities}

We now state our main theoretical results. For this section we restrict attention to the case where the state space is compact. This also covers the finite state case - and hence

\footnotetext{
${ }^{6}$ One additional advantage of the Monte Carlo method centered on (18) is that simulation of the independent consumption processes can be parallelized. This leads to speed gains approaching two orders of magnitude in some of our implementations.
} 


\begin{tabular}{l|rrrrr}
\hline & $m=1000$ & $m=2000$ & $m=3000$ & $m=4000$ & $m=5000$ \\
\hline$n=250$ & 0.9982308 & 0.9982297 & 0.9982308 & 0.9982305 & 0.9982306 \\
& $(0.000025)$ & $(0.000016)$ & $(0.000011)$ & $(0.000012)$ & $(0.000010)$ \\
$n=500$ & 0.9982065 & 0.9982026 & 0.9981934 & 0.9981907 & 0.9981921 \\
& $(0.000028)$ & $(0.000022)$ & $(0.000022)$ & $(0.000028)$ & $(0.000021)$ \\
& 0.9981989 & 0.9981916 & 0.9981917 & 0.9981866 & 0.9981837 \\
& $(0.000037)$ & $(0.000032)$ & $(0.000025)$ & $(0.000025)$ & $(0.000031)$ \\
\hline
\end{tabular}

TABLE 3. Realizations of $\Lambda(m, n)$ when $\Lambda=0.9981498$

any numerical representation of consumption dynamics. For the sake of brevity, most of our exposition focuses on the continuous state case. Translations to the finite state setting are straightforward.

Our interest centers on existence, uniqueness and computability of $V_{t} / C_{t}$ in (1)-(2), although it turns out to be convenient to solve first for

$$
G_{t}:=\left(\frac{V_{t}}{C_{t}}\right)^{1-\gamma}
$$

We use (2), (6) and (20) to rewrite the preference recursion (1) as

$$
G_{t}=\left\{1-\beta+\beta\left\{\mathbb{E}_{t} G_{t+1} \exp \left[(1-\gamma) \kappa\left(X_{t}, X_{t+1}, \varepsilon_{t+1}\right)\right]\right\}^{1 / \theta}\right\}^{\theta}
$$

where

$$
\theta:=\frac{1-\gamma}{1-1 / \psi}
$$

In terms of a stationary Markov solution $G_{t}=g\left(X_{t}\right)$, the restriction in (21) translates to

$$
g(x)=\left\{1-\beta+\beta\left\{\int g(y) \int \exp [(1-\gamma) \kappa(x, y, \varepsilon)] \nu(\mathrm{d} \varepsilon) q(x, y) \mathrm{d} y\right\}^{1 / \theta}\right\}^{\theta}
$$

for all $x \in \mathbb{X}$, where $\nu$ is the distribution of $\varepsilon_{t+1}{ }^{7}$

We solve (22) for the unknown function $g$ by converting it into a fixed point problem. As a first step, we define $K g$ by

$$
K g(x)=\int g(y) \int \exp [(1-\gamma) \kappa(x, y, \varepsilon)] \nu(\mathrm{d} \varepsilon) q(x, y) \mathrm{d} y
$$

\footnotetext{
${ }^{7}$ These transformations utilize homotheticity of Epstein-Zin utility. In particular, existence and uniqueness of $V_{t} / C_{t}$ is equivalent to existence and uniqueness of the wealth-consumption ratio, which is equal to $(1-\beta)^{-1} G_{t}^{1 / \theta}$ in this model.
} 
The action of the linear operator $K$ on $g$ generalizes the idea of applying the matrix $K$ in (11) to a column vector, as per our discussion of the finite state case in section 2.2. Now let $\varphi$ be the scalar function

$$
\varphi(t)=\left\{1-\beta+\beta t^{1 / \theta}\right\}^{\theta}
$$

on $\mathbb{R}_{+} \cdot{ }^{8}$ Then we define $A$ as the operator mapping $g$ into $A g$ where

$$
A g(x)=\varphi(K g(x)) \text {. }
$$

Now (22) can be written as $g(x)=A g(x)$. Thus, fixed points of $A$ coincide with solutions to the recursive utility problem.

Assumption 3.1. The state space $\mathbb{X}$ is compact.

Assumption 3.1 includes the case where $\mathbb{X}$ is finite. It holds for any numerical application but is not always satisfied in the theoretical models we consider. A treatment of the unbounded case is provided in section 6 .

Let $\mathscr{C}$ be the set of positive functions $g$ on $\mathbb{X}$ such that $g\left(X_{t}\right)$ has finite first moment. With $\Lambda$ as defined in (4), we can state our main findings:

Theorem 3.1 (Existence and Uniqueness). If assumptions 2.1 and 3.1 hold, then $\Lambda$ is well defined and the following statements are equivalent:

(a) $\Lambda<1$.

(b) A has a fixed point in $\mathscr{C}$.

(c) There exists a $g \in \mathscr{C}$ such that $\left\{A^{n} g\right\}_{n \geqslant 1}$ converges to an element of $\mathscr{C}$. $^{9}$

(d) A has a unique fixed point in $\mathscr{C}$.

(e) A has a unique fixed point $g^{*}$ in $\mathscr{C}$ and $A^{n} g \rightarrow g^{*}$ as $n \rightarrow \infty$ for any $g$ in $\mathscr{C}$.

From a practical perspective, the most useful result in theorem 3.1 is that a unique solution exists precisely when $\Lambda<1$. Another interesting result is the logical equivalence of (b) and (d), which tells us that at most one solution exists for every parameterization. A third is that, since (c) is equivalent to (e), convergence of successive approximations from any starting point in $\mathscr{C}$ implies that a unique solution exists and this solution is equal to the limit of the successive approximations from every initial condition. Thus, if computing the solution to the model at a given set of parameters is the primary objective, then

\footnotetext{
${ }^{8} \mathrm{We}$ adopt the convention $\infty^{\alpha}=0$ whenever $\alpha<0$ so that, in particular, $\varphi(0)=0$ when $\theta<0$.

${ }^{9}$ Here and below, convergence is in terms of absolute mean error (i.e., $L_{1}$ deviation). Thus, the statement that $g_{n} \rightarrow g$ in $\mathscr{C}$ means that $\int\left|g_{n}(x)-g(x)\right| \pi(x) \mathrm{d} x \rightarrow 0$ as $n \rightarrow \infty$.
} 
convergence of the iterative method itself justifies the claim that the limit is a solution, and that no other solution exists in $\mathscr{C}$.

Condition (a) in theorem 3.1, which translates to $\beta \mathcal{M}_{C}^{1-1 / \psi}<1$, separates the contributions of impatience, intratemporal risk adjustment and intertemporal substitutability of consumption to the valuation of the consumption stream. More impatience (lower $\beta$ ) reduces $\Lambda$, promoting finite valuation. Higher intratemporal risk adjustment $\gamma$ decreases $\mathcal{M}_{C}$ but its impact on $\Lambda$ depends on the elasticity of intertemporal substitution. When preferences are elastic, with $\psi>1$, the income effect of a higher value of future consumption arising from an increase in $\mathcal{M}_{C}$ is stronger than the change in the marginal rate of substitution between current and future consumption. Since $V_{t} / C_{t}$ is denominated in utils per unit of current consumption, it diverges to infinity as $\mathcal{M}_{C}$ increases. The relative strength of the income and substitution effect switches when $\psi<1$, and $\Lambda<1$ is violated when $\mathcal{M}_{C}$ is sufficiently small.

Note that, since the test value $\Lambda$ only depends on consumption through the risk-adjusted long-run mean consumption growth rate $\mathcal{M}_{C}$, transitory details of the consumption process are irrelevant for existence of the continuation value. This insight is used in the applications below, particularly when we compare models with and without learning (sections 4.2 and 4.3 respectively).

The condition $\Lambda<1$ imposes a bound on the average growth rate in the value of longdated consumption strips as their maturity increases. In an IID growth setting, $\log \Lambda$ is exactly equal to this (constant) growth rate, which must be negative for a finite wealthconsumption ratio, and hence the continuation value, to exist. ${ }^{10}$ In other words, the risk adjusted growth rate of consumption $\mathcal{M}_{C}$ has to be sufficiently low relative to the riskfree rate. In more general settings, $\Lambda$ is tied to the principal eigenvalue of a valuation operator used in the pricing of consumption strips. See Borovička et al. (2016) and Qin and Linetsky (2017) for more details on these operators.

The sufficiency component of theorem 3.1 uses fixed point theory for monotone operators, applied to the operator $A$ and focusing on specific existence and uniqueness results originally due to Du (1990). In the introduction we discussed these results briefly, where discussion was limited to the monotone concave case. In fact the results in Du (1990) can handle both monotone concave and monotone convex operators. The operator $A$ always falls into one of these categories, being concave when $\theta<0$ or $\theta \geqslant 1$ and convex otherwise - as follows from the convexity and concavity properties of the function $\varphi$ defined in (24), combined with the linearity of $K$.

\footnotetext{
${ }^{10}$ Section ?? in the online appendix outlines these calculations.
} 
The proof also uses spectral results based around the Krein-Rutman theorem, which extends Perron-Frobenius theory to function spaces. In particular, both the sufficiency and the necessity arguments exploit the spectral radius identity (5) from the introduction, which relies on an extension of a local spectral radius result originally due to V. Ya. Stet'senko. See theorem A.1 in the appendix for the original result, theorem A.2 for the extension and proposition B.4 for the proof of (5).

\section{Applications}

Next we demonstrate how theorem 3.1 can be used to obtain existence and uniqueness of solutions in applied settings. We begin with relatively simple applications and then progress to more realistic specifications of consumption streams.

4.1. Consumption With a Deterministic Time Trend. Consider a model where consumption obeys the geometric trend specification

$$
C_{t}=\tau^{t} X_{t}
$$

where $\tau$ is a positive scalar and $\left\{X_{t}\right\}$ is positive, compactly supported and IID, as in, say, Alvarez and Jermann (2005). Then the risk-adjusted long-run mean consumption growth rate is

$$
\mathcal{M}_{C}=\lim _{n \rightarrow \infty}\left\{\mathcal{R}\left(\frac{\tau^{n} X_{n}}{X_{0}}\right)\right\}^{\frac{1}{n}}=\tau \lim _{n \rightarrow \infty}\left\{\mathbb{E}\left(\frac{X_{n}}{X_{0}}\right)^{1-\gamma}\right\}^{1 /(n(1-\gamma))}=\tau,
$$

where the last equation follows from the fact that the expectation is constant (since the state process is IID). Appealing to theorem 3.1, we find the exact necessary and sufficient condition for a unique solution to exist is

$$
\Lambda=\beta \tau^{1-\frac{1}{\psi}}<1
$$

Since the consumption process (26) is trend-stationary, its stochastic component is immaterial for the long-run distribution of consumption growth. This is why the risk aversion parameter $\gamma$ does not enter into the expression for $\Lambda$ obtained in (28). ${ }^{11}$

\footnotetext{
${ }^{11}$ The absence of the risk aversion parameter in $\Lambda$ contrasts with an earlier condition provided by Alvarez and Jermann (2005), which does depend on $\gamma$. However, our condition $\Lambda<1$ is both necessary and sufficient, from which we can conclude that the risk aversion parameter is indeed irrelevant to existence and uniqueness of a solution. See section 5.2 for more discussion.
} 


\begin{tabular}{l|ccccccc}
\hline parameter & $\mu(1)$ & $\mu(2)$ & $\sigma(1)$ & $\sigma(2)$ & $q(1,1)$ & $q(2,2)$ & $\gamma$ \\
\hline value & 0.007 & 0.0013 & 0.0015 & 0.0063 & 0.93 & 0.83 & 10.0 \\
\hline
\end{tabular}

TABLE 4. Parameter values for the Markov switching model

4.2. Markov Switching Dynamics. To illustrate the finite state case, consider the two state Markov switching specification for consumption growth of Johannes et al. (2016), with

$$
\ln \left(C_{t+1} / C_{t}\right)=\mu\left(X_{t+1}\right)+\sigma\left(X_{t+1}\right) \varepsilon_{t+1}
$$

where $\mathbb{X}=\{1,2\}$ and baseline parameter values as in table 4 . The process $\left\{\varepsilon_{t}\right\}$ is IID and standard normal. We set aside for now the learning component of Johannes et al. (2016) and assume that $X_{t}$ is fully observable. (The learning problem is treated in section 4.3.) The conditions of assumption 2.1 are satisfied with $\ell=1$.

Our first step is to compute $\mathcal{M}_{C}$ for this model using the spectral radius method dicussed in section 2.2. We take $K$ from (11), which in the present setting reduces to the $2 \times 2$ matrix

$$
K(i, j)=K_{i j}=\exp \left[(1-\gamma) \mu(j)+\frac{1}{2}(1-\gamma)^{2} \sigma(j)^{2}\right] q(i, j) .
$$

After inserting the parameters from table 4, we calculate its eigenvalues, evaluate $r(K)$ as the maximal eigenvalue in modulus and then apply $\mathcal{M}_{C}=r(K)^{1 /(1-\gamma)}$ from (16). The result is $\mathcal{M}_{C}=1.005$. Figure 1 shows how $\mathcal{M}_{C}$ varies as $\gamma$ and $\sigma(1)$ shift around their baseline values. Both decrease the risk-adjusted long-run mean consumption growth rate as they rise, with the negative effect of $\gamma$ intensifying as volatility in the good state grows.

With $\mathcal{M}_{C}$ in hand, we can calculate $\Lambda=\beta \mathcal{M}_{C}^{1-1 / \psi}$. With preference parameters $\psi=1.5$ and $\beta=0.998$ from Johannes et al. (2016), we obtain $\Lambda=0.99567$. Hence condition (a) in theorem 3.1 is satisfied and statements (b)-(e) hold true. In particular, a unique and globally attracting solution to the recursive utility problem exists. Conversely, if we (arbitrarily) pair the values $\psi=1.97$ and $\beta=0.999$ from Schorfheide et al. (2018) with this consumption process, then $\Lambda=1.00147$ and, by part (b) of theorem 3.1, no solution exists.

These results turn out to be significant even for the model that does include learning, as the next section details.

4.3. Markov Switching with Learning. Consider again the Markov switching dynamics from the previous section but suppose as in Johannes et al. (2016) that the investor 


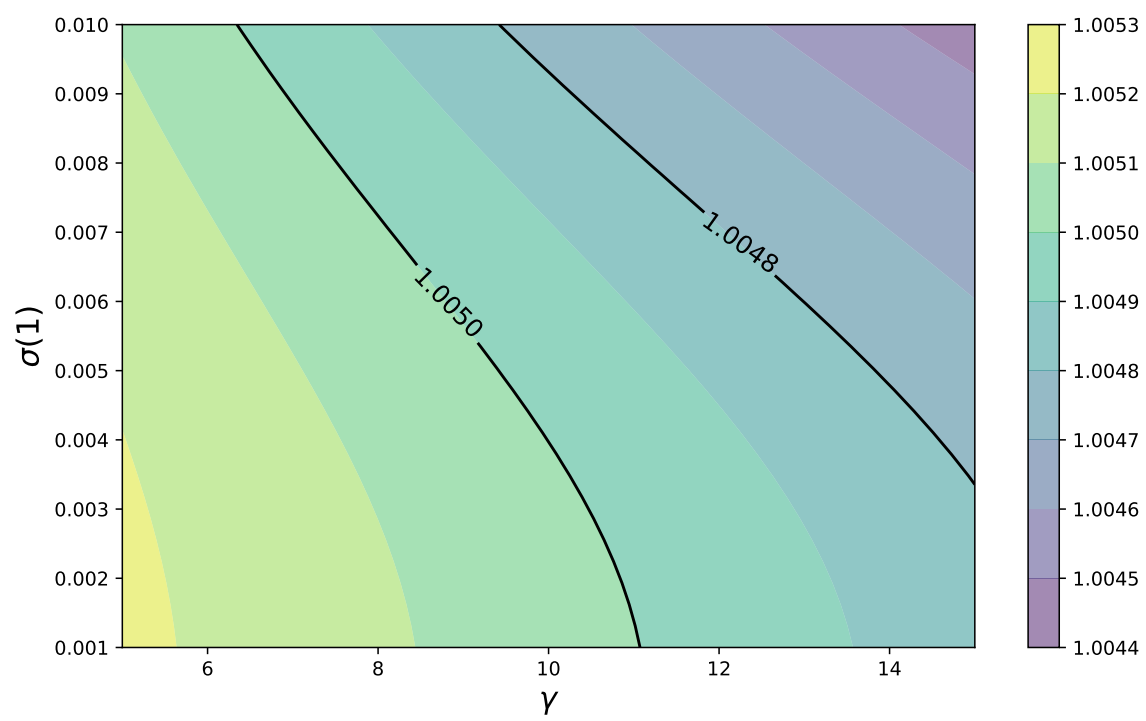

Figure 1. $\mathcal{M}_{C}$ as a function of $\gamma$ and $\sigma(1)$ in the Markov switching model

does not observe the state process $\left\{X_{t}\right\}$. Instead, she forms a Bayesian posterior belief about $X_{t}$ based on her time-0 prior and observations of consumption growth up to time $t$. The investor's filtering problem can be represented recursively, with $\bar{X}_{t} \in[0,1]$ denoting the probability that $X_{t}=1$ under the investor's information set. This new state variable can be shown to follow the law of motion $\bar{X}_{t+1}=h\left(\bar{X}_{t}, Z_{t+1}\right)$, where $Z_{t+1}=\ln \left(C_{t+1} / C_{t}\right)$ is observed consumption growth and

$$
h(x, z)=\frac{[x q(1,1)+(1-x) q(2,1)] \varphi_{1}(z)}{[x q(1,1)+(1-x) q(2,1)]\left(\varphi_{1}(z)-\varphi_{2}(z)\right)+\varphi_{2}(z)} .
$$

Here $\varphi_{j}$ is the density of $Z_{t+1}$ conditional on $X_{t}=j$. Details of the derivation of this standard filtering problem are provided in the online appendix. The matrix $K$ in (30), which served as the valuation operator in the Markov switching problem without learning, is replaced by the operator

$$
\bar{K} g(x)=\int g(y)[\xi(1) y+\xi(2)(1-y)] \bar{q}(x, y) \mathrm{d} y
$$

where

$$
\xi(i)=\exp \left((1-\gamma) \mu(i)+\frac{1}{2}(1-\gamma)^{2} \sigma^{2}(i)\right)
$$


for $i=1,2$ and $\bar{q}$ represents the transition density arising from the subjective belief of the Bayesian learner. In other words, $\bar{q}(x, \cdot)$ is the distribution of $h\left(x, Z_{t+1}\right)$ when $h$ is as defined in $(31)$ and $Z_{t+1}=\ln \left(C_{t+1} / C_{t}\right) \cdot{ }^{12}$

We now evaluate the risk-adjusted mean consumption growth rate (3) under the model with learning, which we denote $\overline{\mathcal{M}}_{C}$, in order to determine the test value $\Lambda$. Denote $\overline{\mathbb{E}}_{t}$ the expectations operator under the investor's time- $t$ information set and $\overline{\mathbb{E}}$ its unconditional version. Hence

$$
\overline{\mathcal{M}}_{C}=\lim _{n \rightarrow \infty}\left\{\overline{\mathbb{E}}\left[\left(C_{n} / C_{0}\right)^{(1-\gamma)}\right]^{\frac{1}{1-\gamma}}\right\}^{1 / n} .
$$

The Law of Iterated Expectations implies that for any $n \in \mathbb{N}$

$$
\min _{X_{0}} \mathbb{E}_{0}\left[\left(C_{n} / C_{0}\right)^{(1-\gamma)}\right] \leqslant \overline{\mathbb{E}}\left[\left(C_{n} / C_{0}\right)^{(1-\gamma)}\right] \leqslant \max _{X_{0}} \mathbb{E}_{0}\left[\left(C_{n} / C_{0}\right)^{(1-\gamma)}\right] .
$$

Raising this pair of inequalities to power $1 /(n(1-\gamma))$ and taking the limit as $n \rightarrow \infty$, we obtain

$$
\lim _{n \rightarrow \infty}\left\{\min _{X_{0}} \mathbb{E}_{0}\left[\left(C_{n} / C_{0}\right)^{(1-\gamma)}\right]^{\frac{1}{1-\gamma}}\right\}^{1 / n} \leqslant \overline{\mathcal{M}}_{C} \leqslant\left\{\max _{X_{0}} \mathbb{E}_{0}\left[\left(C_{n} / C_{0}\right)^{(1-\gamma)}\right]^{\frac{1}{1-\gamma}}\right\}^{1 / n} .
$$

However, the limits of the conditional expectations on the left- and right-hand side of this relationship are independent of the initial state $X_{0}$ due to ergodicity of the twostate Markov chain. Consequently, $\overline{\mathcal{M}}_{C}=\mathcal{M}_{C}$, and the parameters for which unique continuation values exist coincide in the full and partial information models.

This result holds despite the fact that the two models have fundamentally different state spaces and transition densities. The reason for the result is the transitory impact of state uncertainty on the conditional distribution of future consumption growth. Our test value $\Lambda$ depends only on the long-run distribution of consumption growth under the investor's belief. Transitory deviations in the investor's belief about future consumption growth relative to the data generating process, driven by learning about the unobserved state, are inconsequential in the long run and therefore irrelevant for existence of a finite continuation value, despite the fact that they affect the conditional expectations $\overline{\mathbb{E}}_{t}$ in every step of the continuation value recursion (22).

As a consequence, in this class of learning models, existence and uniqueness of the continuation value can be more easily evaluated by computing $\Lambda$ in the full information model as in section 4.2 , which has a simpler state space and transition density.

\footnotetext{
${ }^{12}$ While the normal densities $\varphi_{j}(z)$ specified in Johannes et al. (2016) do not directly imply a transition density $\bar{q}(x, y)$ that satisfies assumption 2.1, we construct in the online appendix an arbitrarily small perturbation of these densities such that assumption 2.1 does hold.
} 


\begin{tabular}{ccccccccc}
$\mu_{c}$ & $\rho$ & $\varphi_{z}$ & $\bar{\sigma}$ & $\varphi_{c}$ & $\rho_{h_{z}}$ & $\sigma_{h_{z}}^{2}$ & $\rho_{h_{c}}$ & $\sigma_{h_{c}}^{2}$ \\
\hline 0.0016 & 0.987 & 0.215 & 0.0035 & 1.0 & 0.992 & 0.0039 & 0.991 & 0.0096
\end{tabular}

TABLE 5. Parameterization of the consumption process with long run risk. Parameter values are posterior median estimates from Schorfheide et al. (2018), Table VII, estimation with consumption and financial markets data.

4.4. Long-Run Risk. Next consider the consumption specification adopted in Schorfheide et al. (2018), where

$$
\begin{gathered}
\ln \left(C_{t+1} / C_{t}\right)=\mu_{c}+z_{t}+\sigma_{c, t} \eta_{c, t+1}, \\
z_{t+1}=\rho z_{t}+\sqrt{1-\rho^{2}} \sigma_{z, t} \eta_{z, t+1}, \\
\sigma_{i, t}=\varphi_{i} \bar{\sigma} \exp \left(h_{i, t}\right) \quad \text { with } \quad h_{i, t+1}=\rho_{h_{i}} h_{i}+\sigma_{h_{i}} \eta_{h_{i}, t+1}, \quad i \in\{c, z\} .
\end{gathered}
$$

The innovations $\left\{\eta_{i, t}\right\}$ and $\left\{\eta_{h_{i}, t}\right\}$ are IID and standard normal for $i \in\{c, z\}$. The state vector associated with these consumption dynamics can be represented as $X_{t}=\left(h_{c, t}, h_{z, t}, z_{t}\right)$. The consumption process parameters used by Schorfheide et al. (2018) are shown in table 5 , while the preference parameters are $\gamma=8.89, \beta=0.999$ and $\psi=1.97$.

All of the conditions of theorem 3.1 hold apart from compactness of the state space, which fails because $X_{t}=\left(h_{c, t}, h_{z, t}, z_{t}\right)$ can take values in all of $\mathbb{R}^{3}$. However, the fact that the correlation coefficients $\rho, \rho_{c}$ and $\rho_{z}$ are less than one in absolute value means that the state space can be compactified by truncating the standard normal shocks $\eta_{z}, \eta_{h_{c}}$ and $\eta_{h_{z}}$. For now this is the path that we pursue. In this compactified setting, theorem 3.1 applies and a unique and globally stable solution exists if and only if $\Lambda<1$.

To evaluate $\Lambda$ at a specific level of truncation, we begin with the Monte Carlo method introduced in section 2.4. This necessitates the use of a random number generator, so the compactification is implemented automatically by truncating the innovations in absolute value to the largest double precision floating point number, without the need to impose further restrictions on the state space. ${ }^{13}$ Table 6 shows summary statistics for 1,000 draws of $\Lambda(m, n)$ as $n$, the time series length for consumption, is varied across the rows, while

\footnotetext{
${ }^{13}$ This evaluates to $2^{53}$, which is $\approx 10^{16}$. A far smaller truncation point would be adequate but there are no obvious benefits to implementing such a modification, since any truncation of the innovations generates a compact state space, regardless of how large. Theorem 3.1 then applies. Further discussion of the effects of truncation is provided below.
} 


\begin{tabular}{cccccccc}
$n$ & mean & std & $\min$ & $25 \%$ & $50 \%$ & $75 \%$ & $\max$ \\
\hline \hline 500 & 0.999408 & 0.000111 & 0.998564 & 0.999385 & 0.999441 & 0.999473 & 0.999529 \\
1000 & 0.999384 & 0.000093 & 0.998551 & 0.999351 & 0.999409 & 0.999446 & 0.999517 \\
1500 & 0.999401 & 0.000061 & 0.999024 & 0.999386 & 0.999421 & 0.999441 & 0.999466 \\
\hline
\end{tabular}

TABLE 6. Descriptive statistics for 1,000 draws of $\Lambda(m, n)$ when $m=5000$

$m$ is held fixed at $1,000 .{ }^{14}$ In all cases, the mean estimate lies close to 0.9994 and the standard deviation in the sample is small. Other values of $m$ and $n$ produce similar numbers. Recalling our earlier finding that $\Lambda(m, n)$ closely approximates $\Lambda$ for moderate choices of $m$ and $n$ (see, e.g., table 3), this leads us to infer with a high degree of certainty that the valuation problem for this compactified model has a unique and globally stable solution.

While the values of $\Lambda(m, n)$ are all close to 1 at the given parameterization of Schorfheide et al. (2018), the outcome $\Lambda<1$ survives reasonably large deviations in the parameters, which further supports the conclusion that the valuation problem is globally stable. Figure 2 illustrates this robustness by presenting the test value $\Lambda$ at neighboring parameterizations. Values are computed by Monte Carlo, with $m=n=1000$. The neighboring parameterizations are obtained by varying two parameters while holding others constant. Figure 2a shows values obtained for $\Lambda$, represented by a contour plot, as $\psi$ and $\mu_{c}$ are varied. Figure $2 \mathrm{~b}$ shows the same as $\beta$ and $\psi$ are varied. Parameterizations to the southwest of the 1.0 contour line are globally stable, while those to the northeast yield $\Lambda>1$ and hence, by part (b) of theorem 3.1, no solution exists. For both subfigures, the Schorfheide et al. (2018) parameterization falls well within the interior of the stable set.

None of the results presented above speak directly to the existence and uniqueness in the original theoretical model, where innovations are not truncated and the state space is unbounded. There are two independent questions that need to be considered here. First, what is the value of $\Lambda$ at the Schorfheide et al. (2018) parameterization when shocks are not truncated? Second, what does the value of $\Lambda$ actually imply for the unbounded state space, given that theorem 3.1 is not applicable?

Neither question is fully answered here, although we can make reasonable conjectures. Regarding the first question, figure 3 studies the impact of truncation of the innovations in this long-run risk model. The truncation value on the horizontal axis is the number

\footnotetext{
${ }^{14}$ See equations (18) and (19) for the definition of $\Lambda(m, n)$. Consumption paths are generated according to (33)-(35), with independent normal variates supplied by a standard random number generator.
} 


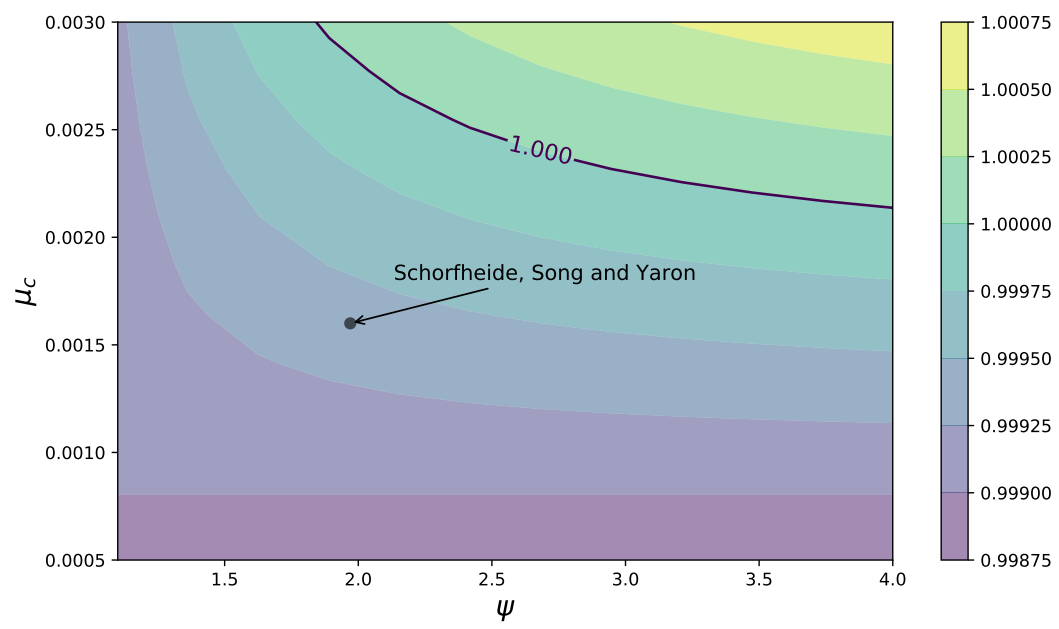

(A) Changes in test value, $\psi$ vs $\mu_{c}$

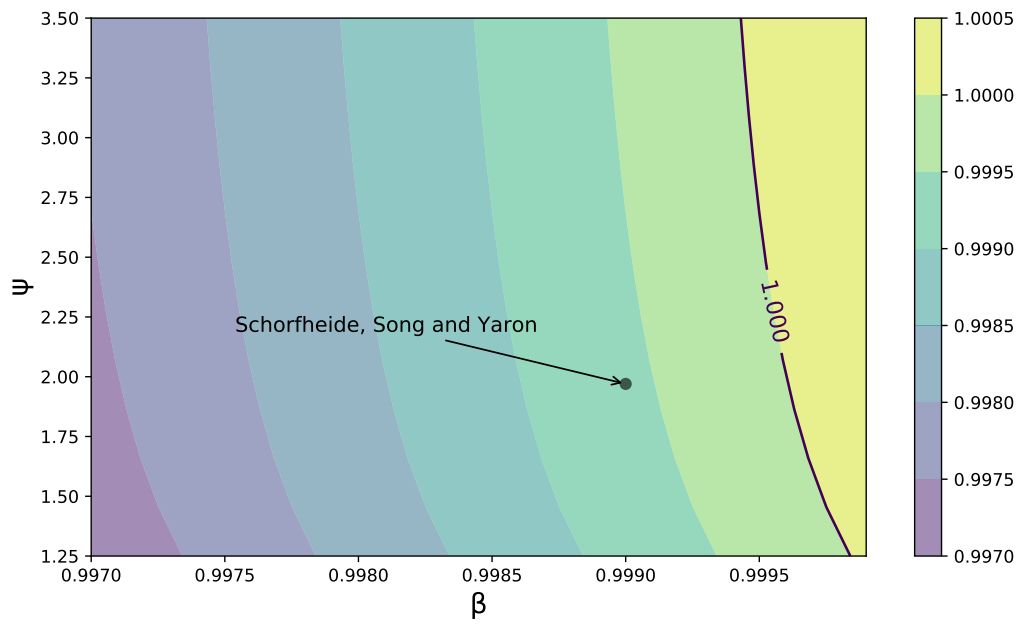

(B) Changes in test value, $\beta$ vs $\psi$

Figure 2. A contour map of $\Lambda$ values near the SSY parameterization

of standard deviations at which the normal shocks in (34) and (35) are truncated. The values on the vertical axis are the corresponding values of $\Lambda(m, n)$, estimated using the Monte Carlo method with $n=m=1000$. The dashed line shows the value of $\Lambda(m, n)$ when no explicit truncation is imposed (although we are working with 64bit floating point numbers, so truncation at around $10^{16}$ standard deviations is implicit). The first point made clear by the figure is that the impact of truncation becomes negligible once the 


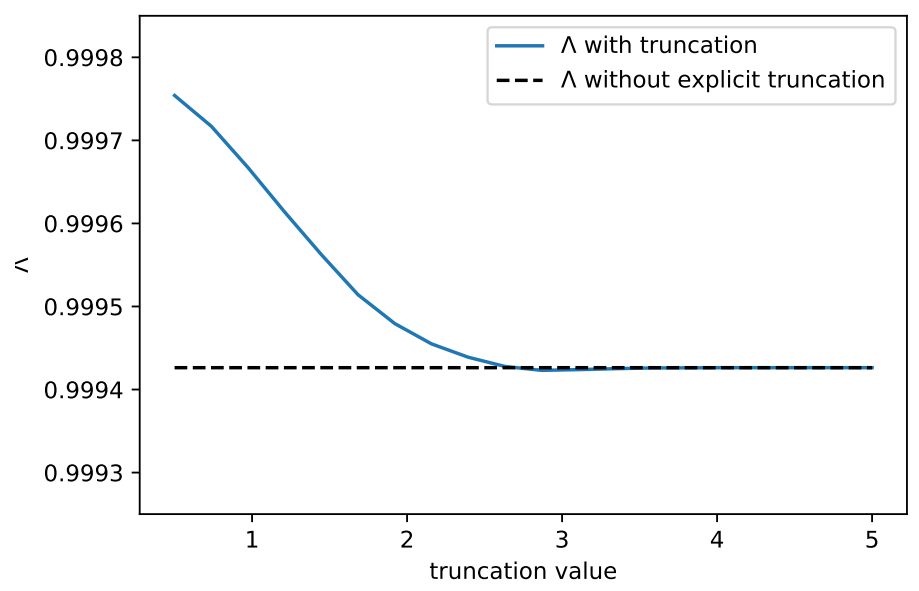

Figure 3. The impact of truncation on $\Lambda$ in the long run risk model

truncation level for the standard normal innovations reaches 3 . This is consistent with our knowledge of the standard normal distribution (i.e., rapidly decreasing tails and $99.7 \%$ of probability mass within three standard deviations of the mean). The second point is that relaxing truncation shifts $\Lambda$ down rather than up. ${ }^{15}$ Thus, there is strong evidence that $\Lambda<1$ holds for the original unbounded model.

The second question asks what does the condition $\Lambda<1$ imply when the state space is not compact and theorem 3.1 cannot be applied. Some guidance in the unbounded setting is provided by theorem 6.1 from section 6 below, which shows that necessity and sufficiency of $\Lambda<1$ for existence extends to the unbounded case. However, theorem 6.1 relies on a side condition on $K$ that is difficult to evaluate in a complex nonlinear model such as the specification of Schorfheide et al. (2018). At the same time, this condition is standard in the literature that uses eigendecompositions to analyze long-run valuations. Section 6 gives more details.

4.5. A Discretized Long-Run Risk Model. A frequently used solution method is to discretize the long-run risk model to a finite state space, since such a model is relatively easy to manipulate and allows for straightforward calculation of all endogenous quantities. We also use the model to compute the equilibrium wealth-consumption ratio at a range

\footnotetext{
${ }^{15}$ This is consistent with expectations because weakening truncation expands the tails of the shocks, increasing volatility. Given the relatively large value of $\gamma$ adopted in Schorfheide et al. (2018), higher volatility tends to decrease the risk-adjusted mean consumption growth rate (see (10) for intuition) and hence $\Lambda$. We tested different combinations of $m$ and $n$ in these calculations but none altered our conclusions.
} 
of parameter values in order to shed light on the asset pricing consequences of moving in the parameter space toward the region where a solution ceases to exist.

Discretization is achieved by applying the Rouwenhorst method to the laws of motion for the state variables (equations (34) and (35)) in each of the three dimensions. The state space is then finite and the Markov chain for the state process is aperiodic and irreducible, so assumptions 2.1 and 3.1 hold. Hence, by theorem 3.1, a unique and globally stable solution exists for the discretized model when $\Lambda<1$. We found this to be true at the baseline parameterization for all levels of discretization we considered. ${ }^{16}$

The contour diagrams in figure 2 suggest that, if we shift the parameters of the model sufficiently far from the baseline setting of Schorfheide et al. (2018), we can find parameterizations where $\Lambda \geqslant 1$. The same is true in the discretized setting. How should we interpret such outcomes? One way to understand failure of existence of a solution to the valuation problem in this model is to study what happens to equilibrium quantities as we move close to the boundary between stability and instability. Consider, for example, table 7 , which shows how the mean wealth-consumption ratio varies with the parameters $\psi$ and $\mu_{c}$, while other parameters are held fixed at the default values in table 5 . The equilibrium wealth-consumption ratio is equal in this model to $(1-\beta)^{-1} g^{*}\left(X_{t}\right)^{1 / \theta}$, where $g^{*}$ is the fixed point of the operator $A$ discussed in theorem 3.1 (see footnote 7 ). The function $g^{*}$ is computed by iterating with $A$ on the arbitrary initial choice $g \equiv 1$, a process for which theorem 3.1 guarantees convergence whenever $\Lambda<1$. $^{17}$

Table 7 covers some of the parameter values explored in figure $2 \mathrm{a}$, which showed that no solution exists when both $\mu_{c}$ and $\psi$ are sufficiently large. For those pairs - or, more precisely, for any pair where $\Lambda \geqslant 1$-we printed the string NA to indicate that no solution exists. For other pairs we printed the mean wealth-consumption ratio. Table 7 shows that, as the parameters approach the boundary between stability and instability, the mean wealth-consumption ratio explodes. This is the nature of nonexistence of solutions: parameters are such that wealth and forward looking valuations are infinite.

\footnotetext{
${ }^{16}$ To handle the stochastic volatility component, we proceed as follows: First we apply the Rouwenhorst method independently to $\left\{h_{c, t}\right\}$ and $\left\{h_{z, t}\right\}$, both of which are linear $\operatorname{AR}(1)$. Then we translate the results into discretized dynamics for $\left\{\sigma_{c, t}\right\}$ and $\left\{\sigma_{z, t}\right\}$, with $H$ and $I$ possible values respectively. Last, for each of the $I$ possible values of $\sigma_{z}$, we again use the Rouwenhorst method to discretize $\left\{z_{t}\right\}$ across $J$ possible states. With $H=I=J=3$, we obtain $\Lambda=0.99944$. For the finer discretizations the value of $\Lambda$ tends to decline, which is consistent with the intuition we obtain from figure 3 .

${ }^{17}$ The calculation was carried out for the discretized representation of the state process, which was in turn computed via multiple iterations of the Rouwenhorst technique, in the manner discussed above. In each case we set $H=I=J=3$, so the state space had 27 elements. Iteration continued until the maximal absolute deviation between successive iterates fell below 1e-6.
} 


\begin{tabular}{ccccccc} 
& $\psi=1.1$ & $\psi=1.68$ & $\psi=2.26$ & $\psi=2.84$ & $\psi=3.42$ & $\psi=4.0$ \\
\hline \hline$\mu_{c}=0.0030$ & 1290.3 & 46604.4 & $\mathrm{NA}$ & $\mathrm{NA}$ & $\mathrm{NA}$ & $\mathrm{NA}$ \\
\hline$\mu_{c}=0.0025$ & 1219.3 & 4610.7 & $4.6 \mathrm{e}+25$ & $\mathrm{NA}$ & $\mathrm{NA}$ & $\mathrm{NA}$ \\
\hline$\mu_{c}=0.0020$ & 1155.7 & 2423.3 & 4986.7 & 12840.7 & 3596674.7 & $1.7 \mathrm{e}+31$ \\
\hline$\mu_{c}=0.0015$ & 1098.4 & 1642.7 & 2142.0 & 2600.6 & 3022.8 & 3412.6 \\
\hline$\mu_{c}=0.0010$ & 1046.5 & 1242.0 & 1362.4 & 1443.9 & 1502.7 & 1547.2 \\
\hline$\mu_{c}=0.0005$ & 999.5 & 998.3 & 998.3 & 998.3 & 998.5 & 998.6 \\
\hline
\end{tabular}

TABLE 7. Mean of the wealth-consumption ratio under the stationary distribution of the state process, across different $\mu_{c}, \psi$ combinations. Monthly consumption in the denominator. As $\psi \rightarrow 1$, the wealth-consumption ratio converges to the constant $(1-\beta)^{-1}=1000$.

4.6. Production Economies. We have so far assumed that consumption follows an exogenously specified process, as given in (6). A large class of models with EpsteinZin preferences studied in the macro-finance literature (Tallarini (2000), Kaltenbrunner and Lochstoer (2010), Croce (2014), and many others) involves endogenously determined consumption processes of the form

$$
\begin{aligned}
C_{t} & =c\left(X_{t}\right) A_{t} \\
\log A_{t+1}-\log A_{t} & =\kappa_{A}\left(X_{t}, X_{t+1}, \varepsilon_{t+1}\right) .
\end{aligned}
$$

For example, suppose we are interested in a production economy where $A_{t}$ is an exogenous technology process with stochastic growth and are solving for an endogenous function $c\left(X_{t}\right)$ that represents stationary deviations from the stochastic trend. Let the assumptions from Section 2 imposed on $X_{t}$ and $\varepsilon_{t}$ hold. Further assume that $c(x)$ is bounded above and away from zero, and denote $\bar{\zeta}=\max _{x_{0}, x_{n}}\left(c\left(x_{n}\right) / c\left(x_{0}\right)\right)^{1-\gamma}$ and $\underline{\zeta}=\min _{x_{0}, x_{n}}\left(c\left(x_{n}\right) / c\left(x_{0}\right)\right)^{1-\gamma}$. In this case

$$
\underline{\zeta} E\left[\left(\frac{A_{n}}{A_{0}}\right)^{1-\gamma}\right] \leqslant E\left[\left(\frac{C_{n}}{C_{0}}\right)^{1-\gamma}\right] \leqslant \bar{\zeta} E\left[\left(\frac{A_{n}}{A_{0}}\right)^{1-\gamma}\right]
$$

and, as a consequence, the risk-adjusted long-run mean consumption growth rate $\mathcal{M}_{C}$ from (3) is given by

$$
\mathcal{M}_{C}=\lim _{n \rightarrow \infty}\left[\mathcal{R}\left(\frac{A_{n}}{A_{0}}\right)\right]^{1 / n} .
$$

We can therefore infer the region of the parameter space for which the endogenously determined continuation value exists directly from the knowledge of the properties of 
the process $A_{t}$, without knowing the details of the function $c(x){ }^{18}$ This is another manifestation of the fact that our condition for existence and uniqueness depends only on long-run properties of the consumption process and transitory details of that process are irrelevant.

\section{Comparisons with Alternative Conditions}

A number of related tests for existence and uniqueness of recursive utilities were discussed in the introduction. In this section we provide a brief comparison of these alternative results with the necessary and sufficient condition $\Lambda<1$.

5.1. Probability One Bounds. Several conditions based on probability one bounds have been proposed in the literature. A representative example is theorem 3.1 of Epstein and Zin (1989), which shows that a solution to the recursive utility problem exists whenever

$$
\psi>1 \text { and } \beta B_{c}^{1-1 / \psi}<1,
$$

where $B_{c}$ is a probability one upper bound on $C_{t+1} / C_{t}$. If such a $B_{c}$ exists, then condition (37) is directly comparable with the condition $\Lambda<1$ because $B_{c}$ always exceeds the risk-adjusted mean consumption growth rate. Indeed, $C_{t+1} / C_{t} \leqslant B_{c}$ for all $t$ implies $C_{n} / C_{0} \leqslant B_{c}^{n}$ for all $n$, and hence, by the definition of $\mathcal{M}_{C}$ in (3),

$$
\mathcal{M}_{C} \leqslant B_{c}
$$

Thus, condition (37) implies $\Lambda<1$. In other words, the condition $\Lambda<1$ is weaker than the condition of Epstein and Zin (1989). Moreover, unless consumption growth is deterministic, the inequality in (38) is strict. This fact recalls the point made earlier that focusing only on the upper tail of the consumption growth distribution leads to overly pessimistic restrictions.

To illustrate, consider the case of the long-run risk model, where consumption growth is as specified in (33). Since consumption growth innovations are normally distributed and have no finite upper bound in this case, we have $B_{c}=+\infty$ and the Epstein-Zin stability coefficient $\beta B_{c}^{1-1 / \psi}$ from condition (37) is also $+\infty$. In contrast, $\Lambda<1$ at the baseline parameters, as discussed in section 4.4 .

\footnotetext{
${ }^{18}$ Another sufficient condition for the result in (36) that is often satisfied in applications is an exponential decay rate in the correlation between $c\left(X_{t+n}\right)^{1-\gamma}$ and $\left(A_{t+1} / A_{t}\right)^{1-\gamma}$ as $n \rightarrow \infty$. The argument can also be further extended to cases when the economy involves additional endogenous nonstationary state variables cointegrated with $A_{t}$, such as aggregate capital. In these cases, the transition law for $X_{t}$ may not satisfy the regularity conditions imposed in Section 2 but such economies can often be regularized using perturbation arguments similar to that described in Section ?? of the online appendix.
} 
5.2. Contraction Arguments. Recall the specification for consumption used in Alvarez and Jermann (2005) and discussed in section 4.1, where $C_{t}=\tau^{t} X_{t}$ with $\tau>0$ and $\left\{X_{t}\right\}$ positive and IID. Let $X_{t}$ have distribution $F$ on $[a, b] \subset \mathbb{R}$ for some positive scalars $a<b$. When consumption obeys (26), Alvarez and Jermann (2005) show that a unique solution to the recursive utility problem exists whenever

$$
\beta \tau^{1-\frac{1}{\psi}} \max _{a \leqslant x \leqslant b}\left\{\int\left(\frac{y}{x}\right)^{1-\gamma} \pi(\mathrm{d} y)\right\}^{\frac{1}{\theta}}<1 .
$$

See Alvarez and Jermann (2005), proposition 9 and lemma A.1 for details.

By way of comparison, we know from section 4.1 that a unique solution exists if and only

if $\beta \tau^{1-\frac{1}{\psi}}<1$. This condition is weaker than (39), since the additional maximized term in (39) always exceeds unity. The difference between the stability conditions arises because the condition from Alvarez and Jermann (2005) enforces contraction in one step. In contrast, the condition $\Lambda<1$ is an asymptotic condition that ignores short-run fluctuations in consumption. This leads to a weaker condition because short-run fluctuations do not impinge on asymptotic outcomes.

\section{Unbounded State Spaces}

Lastly, we return to the setting of theorem 3.1 but now dropping the compactness assumption on the state space (i.e., assumption 3.1) and replacing it with

Assumption 6.1. The operator $K$ is continuous and eventually weakly compact.

Condition 6.1 is similar to assumption 2.1 in Christensen (2017), which is used to study estimation of positive eigenfunctions of valuation operators. An explanation of the terminology and discussion of sufficient conditions can be found in appendix B.

Theorem 6.1. If assumptions 2.1 and 6.1 both hold, then the following statements are equivalent:

(a) $\Lambda<1$.

(b) A has a fixed point in $\mathscr{C}$.

(c) There exists a $g \in \mathscr{C}$ such that $\left\{A^{n} g\right\}_{n \geqslant 1}$ converges to an element of $\mathscr{C}$.

The proof of theorem 6.1 relies heavily on a rather specific spectral continuity result for positive operators obtained by Schep (1980). This continuity is combined with an approximation argument that allows us to bootstrap some results from theorem 3.1. Assumption 6.1 is used to show that the approximation step is valid. 
Compared to theorem 3.1, the calculation of the test value $\Lambda$ in theorem 6.1 is more problematic when no analytical solution exists, as even a flexible method such as Monte Carlo restricts the state space to a finite set of floating point numbers. Moreover, the conclusions are weaker and the technical condition in assumption 6.1 may be nontrivial to test. Nevertheless, the theorem shows us that the core existence result extends to a large class of unbounded theoretical models.

\section{Concluding Remarks}

We derived a simple condition that is both necessary and sufficient for existence and uniqueness of the continuation value and aggregate wealth-consumption ratio in recursive utility models with homothetic Epstein-Zin preferences. Despite the fact that the nonlinear preference recursion intertwines the role of risk aversion and intertemporal elasticity of substitution, our condition separates the two forces. What matters is the average long-run risk-adjusted consumption growth rate and its relationship to intertemporal substitutability of consumption and time discounting. In asset pricing terms, the condition imposes a bound on the long-horizon decay rate of the value of consumption strips as their maturity increases.

We also provided a range of analytical examples, a globally stable method of computing solutions whenever they exist, and numerical methods that allow for efficient evaluation of our condition. Since transitory details of the consumption process do not affect the average long-run risk-adjusted consumption growth rate and hence are irrelevant for our stability condition, insights from this paper can also be applied in production economy settings without explicitly solving the model, as long as cointegration properties of consumption with an exogenous driver of uncertainty can be directly inferred.

While our sharpest results restrict the state space for the underlying Markov state to be compact, we also give extensions to unbounded state spaces and study sensitivity of numerical methods as we relax truncation of the state space. Our results for the unbounded setting are more limited than those for the compact case, but we conjecture that the combination of ever sharper numerical methods and the kinds of theoretical insights provided above forms the most promising road to further understanding of stability and equilibrium properties of asset pricing models used in modern quantitative applications.

\section{Appendix A. General Fixed Point and Spectral Radius Results}

In what follows, the state space $\mathbb{X}$ is allowed to be any compact metric space. In particular, $\mathbb{X}$ can be a compact subset of $\mathbb{R}^{n}$ with the Euclidean distance or an arbitrary finite set 
endowed with the discrete metric $d(x, y)=\mathbb{1}\{x \neq y\}$. Note that, in the latter case, $\kappa$ and $q$ are automatically continuous. The collection of Borel measurable functions $g$ from $\mathbb{X}$ to $\mathbb{R}$ such that $\|g\|:=\int|g| \mathrm{d} \pi<\infty$ is denoted by $L_{1}(\pi)$. Convergence is with respect to $\|\cdot\|$ unless otherwise stated. For $g, h \in L_{1}(\pi)$, the statement $g \leqslant h$ means that $g \leqslant h$ holds $\pi$-almost everywhere, while $g \ll h$ indicates that $g<h$ holds $\pi$-almost everywhere. The symbol $\mathscr{C}$ denotes all $g \in L_{1}(\pi)$ such that $g \gg 0$.

Let $M$ be a linear operator mapping $L_{1}(\pi)$ to itself. The operator norm and spectral radius of $M$ are defined by $\|M\|:=\sup \left\{\|M g\|: g \in L_{1}(\pi),\|g\| \leqslant 1\right\}$ and $r(M):=$ $\lim _{n \rightarrow \infty}\left\|M^{n}\right\|^{1 / n}$ respectively. The operator $M$ is called positive if $M g \geqslant 0$ whenever $g \geqslant 0$. It is called bounded if $\|M\|$ is finite and compact if the image of the unit ball in $L_{1}(\pi)$ under $M$ is precompact in the norm topology. A (possibly nonlinear) operator $S$ mapping a convex subset $E$ of $L_{1}(\pi)$ into itself is called convex on $E$ if $S(\lambda f+(1-\lambda) g) \leqslant$ $\lambda S+(1-\lambda) S g$ for all $f, g \in E$ and all $\lambda \in[0,1]$; and concave if the reverse inequality holds. It is called isotone if $f \leqslant g$ implies $S f \leqslant S g$.

The following is a local spectral radius result suitable for $L_{1}(\pi)$. The proof provided here is due to Mirosława Zima (private communication) and draws on earlier results from Zabreiko et al. (1967).

Theorem A.1 (Zabreiko-Krasnosel'skii-Stetsenko-Zima). Let $h$ be an element of $L_{1}(\pi)$ and let $M$ be a positive compact linear operator. If $h \gg 0$, then

$$
\lim _{n \rightarrow \infty}\left\|M^{n} h\right\|^{1 / n}=r(M) .
$$

Proof of theorem A.1. Let $h$ and $M$ be as in the statement of the theorem. Recall that $r(h, M)=\limsup _{n \rightarrow \infty}\left\|M^{n} h\right\|^{1 / n}$ is the local spectral radius of $M$ at $h$. From the definition of $r(M)$ it suffices to show that $r(h, M) \geqslant r(M)$. To this end, let $\lambda$ be a constant satisfying $\lambda>r(h, M)$ and let

$$
h_{\lambda}:=\sum_{n=0}^{\infty} \frac{M^{n} h}{\lambda^{n+1}} .
$$

The point $h_{\lambda}$ is a well-defined element of $L_{1}(\pi)$ by $\lim \sup _{n \rightarrow \infty}\left\|M^{n} h\right\|^{1 / n}<\lambda$ and Cauchy's root test for convergence. It is also positive $\pi$-almost everywhere, since the sum in (41) includes $h \gg 0$, and since $M$ is a positive operator. Moreover, by standard Neumann series theory (e.g., Krasnosel'skii et al. (2012), theorem 5.1), the point $h_{\lambda}$ also has the representation $h_{\lambda}=(\lambda I-M)^{-1} h$, from which we obtain $\lambda h_{\lambda}-M h_{\lambda}=h$. Because $h \in \mathscr{C}$, this implies that $M h_{\lambda} \leqslant \lambda h_{\lambda}$. Applying this last inequality, compactness of $M$, quasi-interiority of $h_{\lambda}$ and theorem 5.5 (a) of Krasnosel'skii et al. (2012), we must have $r(M) \leqslant \lambda$. Since this inequality was established for an arbitrary $\lambda$ satisfying $\lambda>r(h, M)$, 
we conclude that $r(h, M) \geqslant r(M)$. Hence $r(h, M)=r(M)$. Finally, since $M$ is compact, corollary 1 of Daneš (1987) implies that $r(h, M)=\lim _{n \rightarrow \infty}\left\|M^{n} h\right\|^{1 / n}$, so (40) holds.

The next result is an extension of theorem A.1, which weakens the compactness condition in that theorem while requiring additional positivity.

Theorem A.2. Let $h$ be an element of $L_{1}(\pi)$ and let $M$ be a linear operator on $L_{1}(\pi)$. If $M^{i}$ is compact for some $i \in \mathbb{N}$ and $M f \gg 0$ whenever $f \in L_{1}(\pi)$ and $f \gg 0$, then

$$
\lim _{n \rightarrow \infty}\left\{\int M^{n} h \mathrm{~d} \pi\right\}^{1 / n}=r(M) \text { for all } h \gg 0 .
$$

Proof. Fix $h \in L_{1}(\pi)$ with $h \gg 0$ and choose $i \in \mathbb{N}$ such that $M^{i}$ is a compact linear operator on $L_{1}(\pi)$. Fix $j \in \mathbb{N}$ with $0 \leqslant j \leqslant i-1$. By our assumptions on $M$ we know that $M^{j} h \gg 0$, so theorem A.1 applied to $M^{i}$ with initial condition $M^{j} h$ yields

$$
\left\{\int M^{i n} M^{j} h \mathrm{~d} \pi\right\}^{1 / n}=\left\{\int M^{i n+j} h \mathrm{~d} \pi\right\}^{1 / n} \rightarrow r(M)^{i} \quad(n \rightarrow \infty) .
$$

But $r\left(M^{i}\right)=r(M)^{i}$, so

$$
\left\{\int M^{i n+j} h \mathrm{~d} \pi\right\}^{1 /(i n)} \rightarrow r(M) \quad(n \rightarrow \infty) .
$$

It follows that

$$
\left\{\int M^{i n+j} h \mathrm{~d} \pi\right\}^{1 /(i n+j)} \rightarrow r(M) \quad(n \rightarrow \infty) .
$$

As $j$ is an arbitrary integer satisfying $0 \leqslant j \leqslant i-1$, we conclude that (42) holds.

The next lemma is useful for detecting fixed points in $L_{1}(\pi)$.

Lemma A.3. Let $\left\{g_{n}\right\}$ be a monotone increasing sequence in $L_{1}(\pi)$. If $\left\{g_{n}\right\}$ is bounded above by some $h$ in $L_{1}(\pi)$, then there exists a $g$ in $L_{1}(\pi)$ such that $g_{n} \rightarrow g$. Moreover, if $g_{n}=T^{n} g_{0}$ for some continuous operator $T$ mapping a subset of $L_{1}(\pi)$ to itself, then $g$ is a fixed point of $T$.

Proof. The first claim is a direct consequence of the Monotone Convergence Theorem for integrals. Regarding the second, we have $g_{n} \rightarrow g$ and hence, by continuity, $T g_{n} \rightarrow T g$. But, by the definition of the sequence $\left\{g_{n}\right\}$, we also have $T g_{n} \rightarrow g$. Hence $T g=g$. 


\section{Appendix B. Remaining Proofs, Compact Case}

As before, $q$ is the transition density kernel for $\left\{X_{t}\right\}$. We define $q^{i}$ recursively by $q^{1}=q$ and $q^{i}(x, y)=\int q(x, z) q^{i-1}(z, y) \mathrm{d} z$ for each $x, y \in \mathbb{X}$. By the conditions in section 2, we can take $\ell \in \mathbb{N}$ such that $q^{\ell}>0$, and $\ell$ has this meaning throughout. Also, in all of what remains, we adopt the notation

$$
k(x, y)=\int \exp [(1-\gamma) \kappa(x, y, \varepsilon)] \nu(\mathrm{d} \varepsilon) q(x, y),
$$

so that the operator $K$ satisfies $K g(x)=\int k(x, y) g(y) \mathrm{d} y$. Let $k^{i}$ be defined on $\mathbb{X} \times \mathbb{X}$ by $k^{1}=k$ and $k^{i}(x, y)=\int k(x, z) k^{i-1}(z, y) \mathrm{d} z$. As is easily verified by induction, the $i$-th element $k^{i}$ is the kernel of the $i$-th power of $K$. That is, for all $x \in \mathbb{X}, i \in \mathbb{N}$ and $g \in \mathscr{C}$,

$$
K^{i} g(x)=\int k^{i}(x, y) g(y) \mathrm{d} y .
$$

Throughout this section, assumptions 2.1 and 3.1 are taken to be true.

Lemma B.1. The density $\pi$ is the unique stationary density for $q$ on $\mathbb{X}$. In addition, $\pi$ is everywhere positive and continuous on $\mathbb{X}$.

Proof. Regarding uniqueness, the condition that $q^{\ell}>0$ for some positive $\ell \in \mathbb{N}$ implies irreducibility of $\left\{X_{t}\right\}$, which in turn implies uniqueness of the stationary distribution. See, e.g., Meyn and Tweedie (2009), theorem 10.0.1. ${ }^{19}$ Regarding positivity, suppose to the contrary, there exists a $y$ with $\pi(y)=0$. Since $\pi$ is a stationary density, this means that $\pi(y)=\int q^{\ell}(x, y) \pi(x) \mathrm{d} x=0$. But $q^{\ell}$ is everywhere positive and $\pi$ is a density, and hence positive on a set of positive measure. Thus, the integral must be positive. Contradiction. Finally, take $y_{n} \rightarrow y$ in $\mathbb{X}$ and observe that

$$
\pi\left(y_{n}\right)=\int q\left(x, y_{n}\right) \pi(x) \mathrm{d} x \rightarrow \int q(x, y) \pi(x) \mathrm{d} x=\pi(y)
$$

as $n \rightarrow \infty$. The convergence of the integrals is due to continuity of $q$ and the Dominated Convergence Theorem. The latter can be employed because $q$ is bounded on $\mathbb{X} \times \mathbb{X}$ by compactness of $\mathbb{X}$ and continuity of $q$. Hence $\pi$ is continuous on $\mathbb{X}$.

Lemma B.2. Regarding the operator $K$, the following statements are true:

(a) $K$ is a bounded linear operator on $L_{1}(\pi)$ that maps $\mathscr{C}$ to itself.

(b) $K g$ is continuous at each $g \in \mathscr{C}$.

(c) $K g \neq 0$ whenever $g \in \mathscr{C}$ and $g \neq 0$.

\footnotetext{
${ }^{19}$ To verify irreducibility, pick any $A$ with $\int_{A} \pi(x) \mathrm{d} x>0$. Evidently $A$ has positive Lebesgue measure, so, with $\ell$ chosen so that $q^{\ell}$ is everywhere positive, we have $\int_{A} q^{\ell}(x, y) \mathrm{d} y>0$ for all $x \in \mathbb{X}$. Thus, $A$ is visited with positive probability from any state in $\ell$ steps. In other words, $\pi$-irreducibility holds.
} 
(d) $K g \gg 0$ whenever $g \in \mathscr{C}$ and $g \gg 0$.

Proof. Regarding claim (a), $k$ is continuous and hence bounded by some constant $M$ on $\mathbb{X}$, while $\pi$ is positive and continuous on a compact set, and hence bounded below by some positive constant $\delta$. This yields, for arbitrary $f \in L_{1}(\pi)$,

$$
|K f(x)|=\left|\int k(x, y) f(y) \mathrm{d} y\right| \leqslant M \int \frac{|f(y)|}{\pi(y)} \pi(y) \mathrm{d} y \leqslant \frac{M}{\delta}\|f\| .
$$

It follows directly that $K$ is a bounded linear operator on $L_{1}(\pi)$.

Regarding continuity, fix $g \in \mathscr{C}, x \in \mathbb{X}$ and $x_{n} \rightarrow x$. Using the inequality from (44), we have

$$
k\left(x_{n}, y\right) g(y) \leqslant M \frac{g(y)}{\pi(y)} \pi(y) \leqslant \frac{M}{\delta} g(y) \pi(y) .
$$

Since $g \in L_{1}(\pi)$, the function on the right hand side is integrable with respect to Lebesgue measure, so we can apply the Dominated Convergence Theorem to obtain

$$
\lim _{n \rightarrow \infty} K g\left(x_{n}\right)=\int \lim _{n \rightarrow \infty} k\left(x_{n}, y\right) g(y) \mathrm{d} y=K g(x)
$$

In particular, $K g$ is continuous at any $x \in \mathbb{X}$.

Regarding claim (c), suppose that, to the contrary, we have $K g=0$ for some nonzero $g \in \mathscr{C}$. Let $B=\{g>0\}$. Since $g$ is nonzero, we have $\pi(B)>0$. Since $K g=0$, it must be the case that $\int_{B} k(x, y) \mathrm{d} y=0$ for any $x \in \mathbb{X}$. But then $\int_{B} q(x, y) \mathrm{d} y=0$ for any $x \in \mathbb{X}$. A simple induction argument shows that this extends to the $n$-step kernels, so that, in particular, $\int_{B} q^{\ell}(x, y) \mathrm{d} y=0$ for all $x \in \mathbb{X}$. The last equality contradicts $q^{\ell}>0$, as guaranteed by assumption 2.1.

Part (d) is immediate from $K g(x)=\int g(y) k(x, y) \mathrm{d} y$ and (43).

The next lemma discusses irreducibility of $K$ as a linear operator on $L_{1}(\pi)$. See p. 262 of Meyer-Nieberg (2012) for the definition and further discussion.

Lemma B.3. The operator $K$ is irreducible and $K^{2}$ is compact.

Proof. Consider first irreducibility of $K$. By positivity of $\kappa$ and the condition for irreducibility of kernel operators on p. 262 of Meyer-Nieberg (2012), it suffices to show that, for any Borel set $A$ of $\mathbb{X}$ with $0<\pi(A)<1$, there exists a Borel set $B$ of $\mathbb{X}$ with

$$
0<\pi(B)<1 \text { and } \quad \int_{B} \int_{A} q(x, y) \mathrm{d} x \mathrm{~d} y>0 .
$$


To verify (45), suppose to the contrary that we can choose $A \subset \mathbb{X}$ with $0<\pi(A)<1$ and

$$
\int_{A^{c}} \int_{A} q(x, y) \mathrm{d} x \mathrm{~d} y=\int_{A}\left[\int_{A^{c}} q(x, y) \mathrm{d} y\right] \mathrm{d} x=0 .
$$

Then $\int_{A} \int_{A^{c}} q(x, y) \mathrm{d} y \pi(x) \mathrm{d} x=0$. Hence $A$ is absorbing for $q$, in which case $\pi(A)=1$ by $\pi$-irreducibility of $q$ and proposition 4.2.3 of Meyn and Tweedie (2009). Contradiction.

Regarding compactness, theorem 9.9 of Schaefer (1974) implies that $K^{2}$ will be compact whenever $K$ is weakly compact, which requires that the image of the unit ball $B_{1}$ in $L_{1}(\pi)$ under $K$ is relatively compact in the weak topology. To prove this it suffices to to show that, given $\varepsilon>0$, there exists a $\delta>0$ such that $\int_{A} K|f| \mathrm{d} \pi<\varepsilon$ whenever $f \in B_{1}$ and $\pi(A)<\delta$. This is true because $k$ is continuous and hence bounded on $\mathbb{X}$, yielding

$$
\int_{A} \int k(x, y)|f(y)| \mathrm{d} y \pi(x) \mathrm{d} x=\iint_{A} k(x, y) \pi(x) \mathrm{d} x|f(y)| \mathrm{d} y \leqslant M \pi(A) \int|f(y)| \mathrm{d} y
$$

for some constant $M$. By continuity and positivity of $\pi$ on $\mathbb{X}$ we have $\pi \geqslant a$ for some constant $a>0$, and hence $\int|f(y)| \mathrm{d} y \leqslant a\|f\| \leqslant a$. Hence $\int_{A} K|f| \mathrm{d} \pi<\varepsilon$ whenever $\pi(A)<\varepsilon /(a M)$.

Proposition B.4. $\Lambda$ is well defined and satisfies $\Lambda=\beta r(K)^{1 / \theta}$.

Proof. Since $K^{i}$ is compact for some $i$ and maps positive functions into positive functions (see lemmas B.3 and B.2), we can apply theorem A.2 to $\mathbb{1} \equiv 1$ to obtain $r(K)=$ $\lim _{n \rightarrow \infty}\left\|K^{n} \mathbb{1}\right\|^{1 / n}$. An inductive argument based on (6) shows that, for each $n$ in $\mathbb{N}$, we have $K^{n} \mathbb{1}(x)=\mathbb{E}_{x}\left(C_{n} / C_{0}\right)^{1-\gamma}$. Hence, by the law of iterated expectations,

$$
\left\|K^{n} \mathbb{1}\right\|^{1 / n}=\left\{\mathbb{E}\left(\frac{C_{n}}{C_{0}}\right)^{1-\gamma}\right\}^{1 / n}=\left\{\mathcal{R}\left(\frac{C_{n}}{C_{0}}\right)\right\}^{(1-\gamma) / n} .
$$

Since $r(K)=\lim _{n \rightarrow \infty}\left\|K^{n} \mathbb{1}\right\|^{1 / n}$, this yields

$$
r(K)=\lim _{n \rightarrow \infty}\left\{\mathcal{R}\left(\frac{C_{n}}{C_{0}}\right)\right\}^{(1-\gamma) / n}=\mathcal{M}_{C}^{1-\gamma} .
$$

Because $\theta:=(1-\gamma) /(1-1 / \psi)$, we now have

$$
\beta r(K)^{1 / \theta}=\beta \mathcal{M}_{C}^{1-1 / \psi}=\Lambda .
$$

Theorem B.5. The spectral radius $r(K)$ of $K$ is strictly positive. Moreover, there exists an eigenfunction e of $K$ satisfying

$$
K e=r(K) e \quad \text { and } \quad e \gg 0 .
$$

The function e is continuous everywhere on $\mathbb{X}$. 
Proof. Theorem 4.1.4 and lemma 4.2.9 of Meyer-Nieberg (2012) together with the irreducibility and compactness properties of $K$ obtained in lemma B.3 yield positivity of $r(K)$ and existence of the positive eigenfunction in (47). Lemma B.2 implies that $e$ is continuous, since $e \in \mathscr{C}$ and $e=(K e) / r(K)$.

In what follows, $e$ in (47) is called the Perron-Frobenius eigenfunction.

Since $e$ is positive and continuous, the constants $\underline{e}:=\min _{x \in \mathbb{X}} e(x)$ and $\bar{e}:=\max _{x \in \mathbb{X}} e(x)$ are finite and strictly positive. These facts are now used to study $A$ from (25).

Lemma B.6. Let e be the Perron-Frobenius eigenfunction of $K$. If

$$
\lim _{t \downarrow 0} \frac{\varphi(t)}{t} r(K)>1 \quad \text { and } \quad \lim _{t \uparrow \infty} \frac{\varphi(t)}{t} r(K)<1,
$$

then there exist positive constants $c_{1}<c_{2}$ with the following properties:

(a) If $0<c \leqslant c_{1}$ and $f=c e$, then there exists a $\delta_{1}>1$ such that $A f \geqslant \delta_{1} f$

(b) If $c_{2} \leqslant c<\infty$ and $f=c e$, then there exists a $\delta_{2}<1$ such that $A f \leqslant \delta_{2} f$.

Proof. Let $\lambda:=r(K)$ and let $e$ be the Perron-Frobenius eigenfunction. Let $\underline{e}$ and $\bar{e}$ be the maximum and minimum of $e$ on $\mathbb{X}$, as defined above. Regarding claim (a), observe that, in view of (48), there exists a $\delta_{1}>1$ and an $\varepsilon>0$ such that

$$
\frac{\varphi(t)}{t} \lambda \geqslant \delta_{1} \quad \text { whenever } \quad 0<t<\varepsilon \text {. }
$$

Choosing $c_{1}$ such that $0<c_{1} \lambda \bar{e}<\varepsilon$ and $c \leqslant c_{1}$, we have $c \lambda e(x)<\varepsilon$ for all $x \in \mathbb{X}$, and hence

$$
\operatorname{Ace}(x)=\varphi(c K e(x))=\varphi(c \lambda e(x))=\frac{\varphi(c \lambda e(x))}{c \lambda e(x)} c \lambda e(x) \geqslant \delta_{1} c e(x) .
$$

Turning to claim (b) and using again the hypotheses in (48), we can choose a $\delta_{2}<1$ and finite constant $M$ such that

$$
\frac{\varphi(t)}{t} \lambda \leqslant \delta_{2} \quad \text { whenever } \quad t>M
$$

Let $c_{2}$ be a constant strictly greater than $\max \left\{M /(\lambda \underline{e}), c_{1}\right\}$ and fix $c \geqslant c_{2}$. By the definition of $\underline{e}$ we have $c \lambda e(x) \geqslant c_{2} \lambda \underline{e}>M$ for all $x \in \mathbb{X}$, so

$$
\operatorname{Ace}(x)=\varphi(c \lambda e(x))=\frac{\varphi(c \lambda e(x))}{c \lambda e(x)} \lambda c e(x) \leqslant \delta_{2} c e(x) .
$$

By construction, $0<c_{1}<c_{2}$, so all claims are now established. 
Lemma B.7. If the conditions in (48) hold and $A$ has a fixed point $g^{*}$ in $\mathscr{C}$, then, given any $g \in \mathscr{C}$, there exist functions $f_{1}, f_{2} \in \mathscr{C}$ such that

$$
f_{1} \leqslant A g, g^{*} \leqslant f_{2}, \quad A f_{1} \geqslant f_{1}+\varepsilon\left(f_{2}-f_{1}\right) \quad \text { and } \quad A f_{2} \leqslant f_{2}-\varepsilon\left(f_{2}-f_{1}\right) .
$$

Proof. Fix $g \in \mathscr{C}$. Since $A g$ is continuous and $\mathbb{X}$ is compact, $A g$ attains a finite maximum and strictly positive minimum on $\mathbb{X}$. The same is true of the fixed point $g^{*}=A g^{*}$ and the Perron-Frobenius eigenfunction $e$. Hence, we can choose constants $a_{1}$ and $a_{2}$ such that $0 \ll a_{1} e \leqslant g^{*}, A g \leqslant a_{2} e$. With $a_{1}$ chosen sufficiently small lemma B.6 implies that $A\left(a_{1} e\right) \geqslant \delta a_{1} e$ for some $\delta>1$. Setting $f_{i}:=a_{i} e$, we then have $A f_{1} \geqslant \delta a_{1} e$. Since $\delta>1$, we can write this as $A f_{1} \geqslant a_{1} e+\varepsilon\left(a_{2}-a_{1}\right) e$ for some positive $\varepsilon$. In other words, $A f_{1} \geqslant f_{1}+\varepsilon\left(f_{2}-f_{1}\right)$. The proof of the last inequality is similar.

Theorem B.8. If $\Lambda<1$, then $A$ is globally stable on $\mathscr{C}$.

Proof. First we show that, if $\Lambda<1$, then the conditions in (48) hold. Throughout we use the fact that $\Lambda=\beta r(K)^{1 / \theta}$, as shown in proposition B.4. To start, observe that

$$
\frac{\varphi(t)}{t}=\left\{\frac{1-\beta}{t^{1 / \theta}}+\beta\right\}^{\theta} .
$$

If, on one hand, $\theta<0$, then $\Lambda<1$ implies $\beta^{\theta} r(K)>1$ and, in addition, (50) increases to $\beta^{\theta}$ as $t \rightarrow 0$. Thus, the first inequality in (48) holds. The second inequality also holds because $\varphi(t) / t \rightarrow 0$ as $t \rightarrow \infty$. If, on the other hand, $\theta>0$, then $\beta^{\theta} r(K)<1$ and (50) diverges to $+\infty$ as $t \rightarrow 0$, so the first inequality in (48) holds. The second inequality also holds because $\varphi(t) / t \rightarrow \beta^{\theta}$ as $t \rightarrow \infty$.

To complete the proof of theorem B.8, note that $\varphi$ is either concave or convex, depending on the value of $\theta$. Suppose first that $\varphi$ is concave, which implies that $A$ is both isotone and concave on $\mathscr{C}$. Lemma B.6 yields positive constants $c_{1}<c_{2}$ such that $A c_{1} e \geqslant c_{1} e$ and $A c_{2} e \leqslant c_{2} e$. Theorem 2.1.2 of Zhang (2013), which in turn is based on Du (1990), now implies that $A$ has a fixed point $g^{*} \in L_{1}(\pi)$ satisfying $c_{1} e \leqslant g^{*} \leqslant c_{2} e$. Since $e \gg 0$ and $c_{1}>0$, we have $g^{*} \gg 0$.

Let $g$ be a nonzero element of $\mathscr{C}$. Choose $f_{1}, f_{2}$ as in lemma B.7. Theorem 2.1.2 of Zhang (2013) now implies that every element of $\left[f_{1}, f_{2}\right]$ converges to $g^{*}$ under iteration of $A$. In particular, $A^{n}(A g) \rightarrow g^{*}$ as $n \rightarrow \infty$. But then $A^{n} g \rightarrow g^{*}$ also holds. We conclude that $A$ is globally asymptotically stable on $\mathscr{C}$.

The proof for the convex case is essentially identical.

The next result expands on a line of argument developed by Toda (2018), shifting up to infinite dimensions and allowing $\theta<0$. 
Proposition B.9. If $A$ has a nonzero fixed point in $\mathscr{C}$, then $\Lambda<1$.

Proof. Let $K^{*}$ be the adjoint operator associated with $K$. Since $K$ is irreducible and $K^{2}$ is compact, we can employ the version of the Krein-Rutman theorem presented in lemma 4.2.11 of Meyer-Nieberg (2012). Combining this result with the Riesz Representation Theorem, there exists an $e^{*} \in L_{\infty}(\pi)$ such that

$$
e^{*} \gg 0 \quad \text { and } \quad K^{*} e^{*}=r(K) e^{*} \text {. }
$$

Let $g$ be a nonzero fixed point of $A$ in $\mathscr{C}$. It is convenient in what follows to use the inner product notation $\langle f, h\rangle:=\int f h \mathrm{~d} \pi$ for $f \in L_{1}(\pi)$ and $g \in L_{\infty}(\pi)$.

First consider the case where $\theta<0$, so that, by the definition of $\varphi$ we have $\varphi(t) \leqslant \beta^{\theta} t$ with strict inequality whenever $t>0$. As a result, $g(x)=A g(x)=\varphi(K g(x)) \leqslant \beta^{\theta} K g(x)$, with strict inequality whenever $K g(x)>0$. By part (c) of lemma B.2, we have $K g \geqslant 0$ and $K g \neq 0$. So it must be that $g \leqslant \beta^{\theta} K g$ and $g<\beta^{\theta} K g$ on a set of positive $\pi$-measure. But then, taking $e^{*}$ as in (51), we have $\left\langle e^{*}, \beta^{\theta} K g-g\right\rangle>0$, or, equivalently, $\beta^{\theta}\left\langle e^{*}, K g\right\rangle>\left\langle e^{*}, g\right\rangle$. Using the definition of the adjoint and (51) gives $r(K)\left\langle e^{*}, g\right\rangle=\left\langle K^{*} e^{*}, g\right\rangle=\left\langle e^{*}, K g\right\rangle$, so it must be that $\beta^{\theta} r(K)\left\langle e^{*}, g\right\rangle>\left\langle e^{*}, g\right\rangle$. Hence $\Lambda=\beta^{\theta} r(K)>1$. Because $\theta<0$, this implies that $\Lambda=\beta r(K)^{1 / \theta}<1$.

Next consider the case where $\theta>0$, so that $\varphi(t)>\beta^{\theta} t$ whenever $t>0$. As a result, we have $g(x)=A g(x)=\varphi(K g(x)) \geqslant \beta^{\theta} K g(x)$, with strict inequality whenever $K g(x)>0$. By part (c) of lemma B.2, we have $K g \geqslant 0$ and $K g \neq 0$. So it must be that $g \geqslant \beta^{\theta} K g$ and $g>\beta^{\theta} K g$ on a set of positive $\pi$-measure. But then, taking $e^{*}$ as in (51), we have $\left\langle e^{*}, \beta^{\theta} K g-g\right\rangle<0$, or, equivalently, $\beta^{\theta}\left\langle e^{*}, K g\right\rangle<\left\langle e^{*}, g\right\rangle$. As already shown, we have $r(K)\left\langle e^{*}, g\right\rangle=\left\langle e^{*}, K g\right\rangle$, so it must be that $\beta^{\theta} r(K)\left\langle e^{*}, g\right\rangle<\left\langle e^{*}, g\right\rangle$. Hence $\beta^{\theta} r(K)<1$. Because $\theta>0$, this implies that $\Lambda=\beta r(K)^{1 / \theta}<1$.

Proof of theorem 3.1. Clearly (e) $\Longrightarrow(\mathrm{d})$, which in turn implies (c) since we can take $g$ equal to the fixed point. In addition, (c) implies (b), since $K$ is a bounded linear operator on $L_{1}(\pi)$ and $\varphi$ is continuous on $\mathbb{R}_{+}$, from which it follows that $A$ is continuous on $\mathscr{C}$, and hence any limit of a sequence of iterates $\left\{A^{n} g\right\}_{n \geqslant 1}$ of $A$ is a fixed point of $A$. The implication $(\mathrm{b}) \Longrightarrow(\mathrm{a})$ is due to proposition B.9. Finally, (a) $\Longrightarrow$ (e) by theorem B.8 and proposition B.4.

\section{REFERENCES}

Alvarez, F. and Jermann, U. J. (2005). Using asset prices to measure the persistence of the marginal utility of wealth. Econometrica, 73(6):1977-2016. 
Bansal, R., Kiku, D., Shaliastovich, I., and Yaron, A. (2014). Volatility, the macroeconomy, and asset prices. Journal of Finance, 69(6):2471-2511.

Bansal, R., Kiku, D., and Yaron, A. (2012). An empirical evaluation of the long-run risks model for asset prices. Critical Finance Review, 1(1):183-221.

Bansal, R. and Yaron, A. (2004). Risks for the long run: A potential resolution of asset pricing puzzles. The Journal of Finance, 59(4):1481-1509.

Borovička, J., Hansen, L. P., and Scheinkman, J. A. (2016). Misspecified recovery. Journal of Finance, 71(6):2493-2544.

Christensen, T. M. (2017). Nonparametric stochastic discount factor decomposition. Econometrica, 85(5):1501-1536.

Croce, M. M. (2014). Long-run productivity risk: A new hope for production-based asset pricing? Journal of Monetary Economics, 66:13-31.

Daneš, J. (1987). On local spectral radius. Časopis pro pěstování matematiky, 112(2):177187.

Du, Y. (1990). Fixed points of increasing operators in ordered banach spaces and applications. Applicable Analysis, 38(01-02):1-20.

Epstein, L. G. and Zin, S. E. (1989). Risk aversion and the temporal behavior of consumption and asset returns: A theoretical framework. Econometrica, 57(4):937-969.

Guo, J. and He, X. D. (2018). Recursive utility with investment gains and losses: Existence, uniqueness and convergence. Technical report, SSRN working paper 2790768.

Hansen, L. P., Heaton, J. C., and Li, N. (2008). Consumption strikes back? Measuring long-run risk. Journal of Political Economy, 116(2):260-302.

Hansen, L. P. and Scheinkman, J. A. (2009). Long-term risk: An operator approach. Econometrica, 77(1):177-234.

Hansen, L. P. and Scheinkman, J. A. (2012). Recursive utility in a Markov environment with stochastic growth. Proceedings of the National Academy of Sciences, 109(30):11967-11972.

Johannes, M., Lochstoer, L. A., and Mou, Y. (2016). Learning about consumption dynamics. The Journal of finance, 71(2):551-600.

Kaltenbrunner, G. and Lochstoer, L. A. (2010). Long-run risk through consumption smoothing. Review of Financial Studies, 23(8):3190-3224.

Koopmans, T. C. (1960). Stationary ordinal utility and impatience. Econometrica, pages 287-309.

Krasnosel'skii, M., Vainikko, G., Zabreyko, R., Ruticki, Y., and Stet'senko, V. (2012). Approximate Solution of Operator Equations. Springer Netherlands.

Marinacci, M. and Montrucchio, L. (2010). Unique solutions for stochastic recursive utilities. Journal of Economic Theory, 145(5):1776-1804. 
Meyer-Nieberg, P. (2012). Banach lattices. Springer Science \& Business Media.

Meyn, S. and Tweedie, R. L. (2009). Markov chains and stochastic stability. Cambridge University Press.

Qin, L. and Linetsky, V. (2017). Long-term risk: A martingale approach. Econometrica, 85(1):299-312.

Rouwenhorst, K. G. (1995). Asset pricing implications of equilibrium business cycle models. In Frontiers of Business Cycle Research, pages 294-330. Princeton University Press.

Schaefer, H. (1974). Banach Lattices and Positive Operators. Berlin-Heidelberg, New York.

Schep, A. (1980). Positive diagonal and triangular operators. Journal of Operator Theory, pages $165-178$.

Schorfheide, F., Song, D., and Yaron, A. (2018). Identifying long-run risks: A bayesian mixed-frequency approach. Econometrica, 86(2):617-654.

Tallarini, T. D. (2000). Risk-sensitive real business cycles. Journal of Monetary Economics, 45(3):507-532.

Toda, A. A. (2018). Wealth distribution with random discount factors. Journal of Monetary Economics.

Weil, P. (1990). Nonexpected utility in macroeconomics. The Quarterly Journal of Economics, 105(1):29-42.

Zabreiko, P., Krasnosel'skii, M., and Stetsenko, V. Y. (1967). Bounds for the spectral radius of positive operators. Mathematical Notes, 1(4):306-310.

Zhang, Z. (2013). Variational, topological, and partial order methods with their applications. Springer. 